\title{
NUMERICAL SCHEMES OF DIFFUSION ASYMPTOTICS AND MOMENT CLOSURES FOR KINETIC EQUATIONS
}

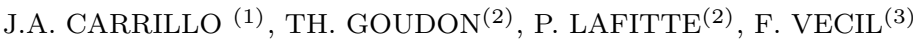 \\ Abstract. We investigate different models that are intended to de- \\ scribe the small mean free path regime of a kinetic equation, a partic- \\ ular attention being paid to the moment closure by entropy minimiza- \\ tion. We introduce a specific asymptotic-induced numerical strategy \\ which is able to treat the stiff terms of the asymptotic diffusive regime. \\ We evaluate on numerics the performances of the method and the abil- \\ ities of the reduced models to capture the main features of the full \\ kinetic equation.
}

\section{INTRODUCTION}

We are interested in numerical simulations of "intermediate" models for kinetic equations in diffusion regimes. Such questions arise in many application fields where we adopt a statistical description of a large set of "particles": neutron transport in nuclear engineering, radiative transfer, rarefied gas dynamics... The unknown is the particle distribution function that gives the number of particles being at time $t$ and position $x$ in a certain physical state described by the variable $v$. In most of the applications, $v$ is nothing but the translational velocity, or the direction of flight of the particles and, assuming that $v$ belongs to a certain measured set $(V, \mathrm{~d} \mu)$, the quantities of interest are essentially averages over $v$ of the unknown. The evolution of the particles obeys the following equation

$$
\varepsilon \partial_{t} f_{\varepsilon}+v \partial_{x} f_{\varepsilon}=\frac{1}{\varepsilon} Q\left(f_{\varepsilon}\right) .
$$

In the right hand side, the operator $Q$ is intended to describe the interactions that particles are subject to; the dimensionless parameter $\varepsilon>0$ is related to the mean free path, that is the average distance travelled by the particles without being subject to any interaction. As $\varepsilon \rightarrow 0$ the unknown $f_{\varepsilon}$ relaxes to an equilibrium the dependence of which with respect to $v$ is

1991 Mathematics Subject Classification. 65M05, 35Q99, 35B25, 82C70, 82-08.

Key words and phrases. Diffusion Asymptotics. Moment Closure. Hyperbolic Systems. Asymptotic Preserving Schemes. 
fixed and the dynamics is described by the evolution of only macroscopic quantities. As we shall see below, it turns out that under some suitable assumptions on the collision operator $Q$, the limit equation reduces to a mere diffusion equation. However, for applications, one is interested in preserving more information concerning the microscopic setting that motivates the derivation of reduced $\varepsilon$-dependent models. Then, it is legitimate to address the following two-fold question: Is the reduced model consistent to the diffusion approximation? How accurate is the obtained approximation and in which sense is it better than the solution of the limit diffusion equation?

In this paper we investigate numerically these questions, restricting to the simplest situation. Namely, we only deal with the one-dimensional framework $(x \in \mathbb{R}, v \in V \subset \mathbb{R})$ and the collision operator is a mere relaxation operator

$$
Q(f)=\int_{V} f \mathrm{~d} \mu(v)-f .
$$

Throughout the paper $(V, \mathrm{~d} \mu)$ is required to satisfy

$$
\left\{\begin{array}{l}
\int_{V} \mathrm{~d} \mu(v)=1, \\
\text { for any odd integrable function } h: V \rightarrow \mathbb{R}, \quad \int_{V} h(v) \mathrm{d} \mu(v)=0, \\
\int_{V} v^{2} \mathrm{~d} \mu(v)=d \quad \text { is positive. }
\end{array}\right.
$$

Typical examples are therefore:

- $V=(-1,+1)$ endowed with the normalized Lebesgue measure,

- $V=\left\{v^{1}, \ldots, v^{M}\right\}$ where the $v^{i}$ 's are well-chosen points in $(-1,+1)$, endowed with the discrete velocity measure, $\mathrm{d} \mu(v)=$ $=\frac{1}{M} \sum_{i=1}^{M} \delta\left(v=v^{i}\right)$,

- $V=\mathbb{R}$ endowed with the Gaussian measure $\mathrm{d} \mu(v)=(2 \pi)^{-1 / 2} e^{-v^{2} / 2} \mathrm{~d} v$.

Under these hypotheses, as we shall recall below, the behavior of $f_{\varepsilon}$ for small $\varepsilon$ 's is given by the heat equation

$$
\partial_{t} \rho-d \partial_{x x}^{2} \rho=0 .
$$

Looking at such a simple situation makes a direct computation of the solution $f_{\varepsilon}$ affordable, including for small values of $\varepsilon$. Therefore we have data at hand to compare with the solutions of reduced models. However, evaluating $f_{\varepsilon}$ when $\varepsilon$ is small has a high numerical cost. Thus, it does not make sense to extend it to several dimensions for our purposes. Nevertheless, we can 
take advantage of our understanding of the limit process to design a numerical method that is well-suited to the asymptotic regime. The scheme we analyze is based on a splitting strategy with a convective-like step involving $\mathcal{O}(1)$ speeds and an explicitly solvable ODE step containing stiff sources. Hence, the scheme, which is naturally asymptotic preserving, is amenable to a fully explicit treatment, free of any $\varepsilon$-dependent restriction, and provides accurate results for a quite cheap numerical cost. Another viewpoint consists in using reduced macroscopic models which are intended to reproduce the main features of the original equation (1). Usually these models are derived either by using some truncated Chapman-Enskog expansion or by imposing a closure to the system that is satisfied by some moments of $f_{\varepsilon}$. A crucial requirement that is usually addressed to the model is to satisfy the so-called limited-flux property. In what follows a particular attention will be paid to the model the derivation of which relies on the Entropy Minimization Principle. In itself the numerical simulation of the reduced models is an issue, due to the presence of stiff terms and large speeds of propagation, that depend on $\varepsilon$. Nevertheless, we introduce original specific schemes for these models using relaxation techniques that we treat following the numerical philosophy evoked above, and interpreting the relaxing system as a discrete kinetic equation. This approach allows to compute efficiently the solutions of the macroscopic models.

The paper is organized as follows, postponing references to the existing literature to the following Sections. In Section 2, we recall some basic facts on the diffusion asymptotics and we present the reduced models we are interested in. In Section 3, we detail the derivation of the asymptoticallyinduced scheme for (1)-(2). We discuss the splitting strategy as well as the numerical boundary conditions which are designed to satisfy the mass conservation. Section 4 is devoted to adapting the method to the macroscopic models. This relies on the interpretation of the models through a relaxation limit. We end with the discussion of the numerical results in Section 5, with in particular simulations of the traditional Su-Olson benchmark.

\section{A Brief Overview on Diffusion Asymptotics and Moment Closures}

2.1. Diffusion Limit. We check readily that Assumption (3) has the following remarkable consequences.

Lemma 1 (Dissipation Properties of the Collision Operator). Assume (3). Then the operator defined by (2) satisfies

i) $Q$ is a bounded operator on $L^{p}(V), 1 \leq p \leq \infty$ spaces; 
ii) $Q$ is conservative which means that for any $f \in L^{1}(V, \mathrm{~d} \mu)$,

$$
\int_{V} Q(f) \mathrm{d} \mu(v)=0 .
$$

iii) $Q$ satisfies the dissipation property

$$
-\int_{V} Q(f) f \mathrm{~d} \mu(v)=\int_{V}|f-\langle f\rangle|^{2} \mathrm{~d} \mu(v) \geq 0,
$$

for any $f \in L^{2}(V, \mathrm{~d} \mu)$, where the bracket is a shortcut notation for the average over $V$;

iv) The elements of the kernel of $Q$ are independent of the microscopic variable $v: \operatorname{Ker}(Q)=\operatorname{Span}(\mathbb{1})$;

v) The following Fredholm alternative holds: for any $h \in L^{2}(V)$ satisfying $\langle h\rangle=0$, there exists a unique $f \in L^{2}(V)$ such that $Q(f)=h$ and $\langle f\rangle=0$.

The Fredholm alternative follows from a direct application of the LaxMilgram theorem applied to the variational formula $\int_{V} Q(f) g \mathrm{~d} \mu(v)=$ $=\int_{V} h g \mathrm{~d} \mu(v)$ on the closed subspace $\left\{f \in L^{2}(V),\langle f\rangle=0\right\}$.

As $\varepsilon$ tends to 0 , the number of interactions or "collisions" events per time unit increases. Accordingly, we can expect for small $\varepsilon$ 's that $f_{\varepsilon}$ resembles an element of the kernel of the operator $Q$ :

$$
f_{\varepsilon}(t, x, v) \simeq \rho(t, x),
$$

and it remains to describe the evolution of the macroscopic quantity $\rho$. The asymptotics can be readily understood by inserting the following Hilbert expansion

$$
f_{\varepsilon}=F_{0}+\varepsilon F_{1}+\varepsilon^{2} F_{2}+\ldots
$$

into (1). Identifying terms arising with the same power of $\varepsilon$, we obtain

- At the leading order $Q\left(F_{0}\right)=0$ that confirms $F_{0}=\rho(t, x)$,

- Next, we have $Q\left(F_{1}\right)=v \partial_{x} F_{0}$. Then, we appeal to the second condition in (3) (applied with $h(v)=v$ ) which allows to make use of the Fredholm alternative. Accordingly, for the simple operator (2), we get $F_{1}(t, x, v)=-v \partial_{x} \rho(t, x)$.

- Then, we obtain a closed equation for $\rho$ by integrating over $v$ the relation: $Q\left(F_{2}\right)=\partial_{t} F_{0}+v \partial_{x} F_{1}$. We obtain

$$
\partial_{t} \rho+\partial_{x}\left(\int_{V}\left(-v^{2} \partial_{x} \rho\right) \mathrm{d} \mu(v)\right)=0
$$

that is the diffusion equation (4) for $\rho$. 
Remark 1 (Time Scaling). The time scaling in (1) is motivated by the fact, embodied into (3), that the equilibrium functions, i.e. the elements of $\operatorname{Ker}(Q)$, have a vanishing flux: considering only the penalization of the collision term, we would be led to the uninspiring equation $\partial_{t} \rho=0$.

The convergence of $f_{\varepsilon}$, solution of (1), to $\rho$, solution of (4), has been widely investigated under various and general assumptions, including non linear situations motivated by physical applications; we refer among others to $[2,3,15,26,22,40,27]$. Under suitable regularity assumptions, we can make the Hilbert expansion approach rigorous, estimate the remainder and justify the convergence with a rate. We refer to [3] for the following statement, which is part of the folklore in kinetic theory.

Theorem 1 (Asymptotic Convergence Rate). Assume that (3) hold. Let $\bar{\rho}>0$ be a constant. Let $f_{0}: \mathbb{R} \times V \rightarrow \mathbb{R}$ such that $f_{0}-\bar{\rho} \in L^{2}(\mathbb{R} \times V)$.

i) Then, as $\varepsilon$ goes to $0, f_{\varepsilon}$ and $\rho_{\varepsilon}$ converge to $\rho$ strongly in $L_{\text {loc }}^{2}\left(\mathbb{R}^{+} \times\right.$ $\mathbb{R})$, and $\rho_{\varepsilon}$ converges to $\rho$ in $\mathcal{C}\left([0, T] ; L^{2}(\mathbb{R})\right.$ - weak $)$, where $\rho$ is the solution to the heat equation (4) with initial datum $\rho_{\mid t=0}=$ $\int_{V} f_{0}(x, v) d \mu(v)$.

ii) If the initial datum is close to a smooth enough macroscopic state, say e.g. $\left\|f_{0}-\rho_{0}\right\|_{L^{2}(\mathbb{R} \times V)} \leq \varepsilon$, with $\left(\rho_{0}-\bar{\rho}\right) \in H^{3}(\mathbb{R})$, then, for any $0<T<\infty$, there exists $C_{T}>0$ such that one has

$$
\left\|f_{\varepsilon}-\rho\right\|_{L^{2}((0, T) \times \mathbb{R} \times V)} \leq C_{T} \varepsilon .
$$

2.2. Approximate Models. We are interested in intermediate models, which are intended to be in between the full kinetic equation (1) and the heat equation (4). Such models are expected to provide "better" approximations of $f_{\varepsilon}$ for moderate values of $\varepsilon$, that are small, but possibly not so small. We also expect that such a model retains more information from the microscopic modelling and we address the question of "how close" to the original unknown $f_{\varepsilon}$ the approximate solution is. Finally, from a practical viewpoint, one should expect that the solution of the intermediate model can be computed with a reduced computational cost. Of course, the solution $\rho$ of (4) already provides an approximation of order $\mathcal{O}(\varepsilon)$ in $L^{2}$ norm, but it has the drawback of loosing completely any microscopic feature since it does not depend on $v$. It could also be tempting to use as an approximation the Hilbert expansion truncated at first order, the so-called $\mathbb{P} 1$ approximation

$$
f_{\varepsilon}(t, x, v) \simeq \rho(t, x)-\varepsilon v \partial_{x} \rho(t, x)
$$

with $\rho$ still the solution of (4). However, such an approximation is not non negative for any $t, x, v$. Furthermore, the heat equation propagates information at infinite speed while in (1) characteristic speeds are of order $\mathcal{O}(1 / \varepsilon)$, at least if the set of velocities is bounded. Actually, finite speed of 
propagation and preservation of non-negativeness are related; indeed, since $f_{\varepsilon} \geq 0$, we have the following relation between the macroscopic current and density

$$
\left|\int_{V} \frac{v}{\varepsilon} f_{\varepsilon} \mathrm{d} \mu(v)\right| \leq \int_{V} \frac{|v|}{\varepsilon} f_{\varepsilon} \mathrm{d} \mu(v) \leq \frac{\|v\|_{L^{\infty}(V)}}{\varepsilon} \int_{V} f_{\varepsilon} \mathrm{d} \mu(v) .
$$

Therefore, we can address that a suitable approximation fulfills this so called "limited flux condition", which is thus guaranteed for free if the approximation is non negative.

To obtain intermediate models, a general strategy consists in writing a system of equations defined by the evolution of moments of $f_{\varepsilon}$. The system is not closed since the convection term makes the $(k+1)$-th moment appear in the evolution equation of the $k$ th moment. Then, we impose a relation between the higher moment involved in the system and the previous ones. We expect that this closure provides a suitable approximation of the evolution of the kinetic density. For (1), it is enough to consider the evolution of the zeroth and first order moments. Let us set

$$
\left(\begin{array}{c}
\rho_{\varepsilon} \\
J_{\varepsilon} \\
\mathbb{P}_{\varepsilon}
\end{array}\right)=\int_{V}\left(\begin{array}{l}
1 \\
v / \varepsilon \\
v^{2}
\end{array}\right) f_{\varepsilon} \mathrm{d} \mu(v) .
$$

We get the mass conservation

$$
\partial_{t} \rho_{\varepsilon}+\partial_{x} J_{\varepsilon}=0
$$

completed by

$$
\varepsilon^{2} \partial_{t} J_{\varepsilon}+\partial_{x} \mathbb{P}_{\varepsilon}=-J_{\varepsilon}
$$

According to [13], we are interested in two possible closure strategies:

(C1) Either we define an approximation, formally close to the $\mathbb{P} 1$ formula, but which preserves non negativity. By using this approximation into the conservation law (6), we obtain a possibly nonlinear equation, that, in some sense, interpolates between transport and diffusion.

(C2) Or we close the moment system (6)-(7), so that we obtain a hyperbolic system that restores the finite speeds of propagation.

We refer to [13] and the references therein for further detail. Let us introduce the following notation

$$
\mathbb{F}(\beta)=\int_{V} e^{\beta v} \mathrm{~d} \mu(v), \quad \mathbb{G}(\beta)=\frac{\mathbb{F}^{\prime}}{\mathbb{F}}(\beta)
$$

and

$$
\psi(u)=\frac{\mathbb{F}^{\prime \prime}}{\mathbb{F}}\left(\mathbb{G}^{(-1)}(u)\right) .
$$


The zeroth order closure (C1) is based on the modified Hilbert expansion

$$
f_{\varepsilon}=\exp \left(a_{0}+\varepsilon a_{1}+\varepsilon^{2} a_{2}+\ldots\right)
$$

Truncating at first order, we get the approximation

$$
\tilde{f}_{\varepsilon}(t, x, v)=\frac{\varrho(t, x)}{Z(t, x)} \exp \left(-\varepsilon v \frac{\partial_{x} \varrho}{\varrho}(t, x)\right),
$$

with $Z(t, x)$ to normalize the density to $\varrho(t, x)$. Plugging this expression into the moment equation, $\varrho$ satisfies

$$
\partial_{t} \varrho-\partial_{x}\left(\frac{\varrho}{\varepsilon} \mathbb{G}\left(\varepsilon \frac{\partial_{x} \varrho}{\varrho}\right)\right)=0 .
$$

The first order closure (C2) follows from a Entropy Minimization Principle. This idea is due to Levermore [33, 34, 37, 35, 36], but it also appears in various physical applications $[12,19]$. It works as follows. For given $\varrho, J$, let

$$
\tilde{f}=\operatorname{argmin}\left\{\int_{V} f \ln (f) \mathrm{d} \mu(v), \quad \int_{V}(1, v / \varepsilon) f \mathrm{~d} \mu(v)=(\varrho, J)\right\} .
$$

We obtain

$$
\widetilde{f}(v)=e^{\lambda_{0}+\lambda_{1} v / \varepsilon}
$$

where the Lagrange multipliers $\lambda_{0,1}$ are defined by the constraints

$\varrho=\int_{V} e^{\lambda_{0}+\lambda_{1} v / \varepsilon} \mathrm{d} \mu(v)=e^{\lambda_{0}} \mathbb{F}\left(\lambda_{1} / \varepsilon\right), \quad J=\int_{V} \frac{v}{\varepsilon} e^{\lambda_{0}+\lambda_{1} v / \varepsilon} \mathrm{d} \mu(v)=\frac{\rho}{\varepsilon} \mathbb{G}\left(\lambda_{1} / \varepsilon\right)$.

Then, we use $\widetilde{f}$ to define the second moment that closes the system (6)-(7). Namely, we set

$$
\mathbb{P}=\int_{V} v^{2} \widetilde{f}(v) \mathrm{d} \mu(v)=\varrho \frac{\mathbb{F} "}{\mathbb{F}}\left(\lambda_{1} / \varepsilon\right)=\varrho \psi(\varepsilon J / \varrho),
$$

and we are thus led to the system

$$
\left\{\begin{array}{l}
\partial_{t} \varrho+\partial_{x} J=0, \\
\varepsilon^{2} \partial_{t} J+\partial_{x}(\varrho \psi(\varepsilon J / \varrho))=-J .
\end{array}\right.
$$

The microscopic approximation is defined by

$$
\widetilde{f}_{\varepsilon}(t, x, v)=\varrho(t, x) \frac{\exp \left[v \mathbb{G}^{(-1)}(\varepsilon J / \varrho(t, x))\right]}{\mathbb{F} \circ \mathbb{G}^{(-1)}(\varepsilon J / \varrho(t, x))} .
$$

Of course, Equations (8) and (9) highly depend on the considered measure $\mathrm{d} \mu$ through the functions $\mathbb{F}, \mathbb{G}$ and $\psi$ :

- For the Lebesgue measure, we have $\mathbb{F}(\beta)=\sinh (\beta) / \beta, \mathbb{G}(\beta)=$ $\operatorname{coth}(\beta)-1 / \beta$. 
- For the discrete 2-velocity measure, we have $\mathbb{F}(\beta)=\cosh (\beta)$. The first order closure (9) is in this case completely equivalent to the original kinetic model and there is no approximation at all.

- For the Gaussian measure, we have $\psi(u)=1+u^{2}$. The zeroth order closure actually leads to the heat equation, and the first order closure gives the isothermal Euler system.

In [13], the well-posedness of (8) and (9) is justified, at least for small and smooth initial data, but, hopefully, with an $\varepsilon$-free smallness condition. (We also refer to [14] for preliminary discussions on weak solutions.) Furthermore it is shown that $\left\|f_{\varepsilon}-\widetilde{f}_{\varepsilon}\right\|_{L^{2}}$ is of order $\mathcal{O}(\varepsilon)$. This estimate is a bit disappointing since it is not better than those evaluating the distance to the solution of the heat equation. Our aim in this paper is to investigate numerically (1)-(2) and its approximation (8) or (9)-(10), compared to the heat equation and the $\mathbb{P} 1$ approximation. It is indeed interesting to check numerically whether we can expect sharper estimates or not. It is also important in view of applications to discuss how the quality of the approximation is degraded as $\varepsilon$ increases and to know if one of the approximation strategies has some decisive advantages. Let us mention that there exist a huge variety of possible closure methods, based either on mathematical arguments or physical grounds, and we mention among others [37, 11].

\section{Asymptotic Preserving Explicit Kinetic Scheme}

On the numerical viewpoint, the computation of (1)-(2) is also a challenging question due to the presence of large, say $\mathcal{O}(1 / \varepsilon)$, speeds of propagation and stiff terms. An attempt to solve (1)-(2) by integrating the equation along the characteristics following a splitting strategy between collisions and transport through lines $x+t v / \varepsilon$ fails for small $\varepsilon$. Since in general the characteristics do not end at a point of the discrete mesh, this approach needs to be completed by a suitable interpolation procedure. It gives rise to semi-lagrangian numerical methods that have been used successfully for Vlasov's like equations $[17,18]$. Proceeding naively, such a procedure can produce unacceptable numerical diffusion. One can repair this drawback by using interpolation procedures based on the WENO approach. We refer to $[47,46]$ for the basis of the WENO method, and to [10] for a description of the adaptation to design an accurate interpolation method. Of course, for small $\varepsilon$ 's these computations become unbearably time consuming with large meshes and small time step due to the large velocities that are involved.

Asymptotic schemes working in the stiffness regime have to be developed. We propose an alternative approach using a splitting scheme inspired by the Hilbert expansion that treats the stiffness of (1). The method is well fitted and much less costly than the previous approach to the diffusion regime 
while remaining fully explicit. This numerical method, which improves the scheme already proposed in [21], is a fully explicit variation of the methods introduced in [30,31], and it has successfully been used in other contexts $[21,9,28]$. The scheme is based on the expansion

$$
f_{\varepsilon}=\rho_{\varepsilon}+\varepsilon g_{\varepsilon}, \quad \rho_{\varepsilon}(t, x)=\int_{V} f_{\varepsilon} \mathrm{d} \mu(v),
$$

where the dissipation properties of the operator $Q$ imply that the "fluctuations" $g_{\varepsilon}$ are indeed bounded in $L^{2}\left(\mathbb{R}^{+} \times \mathbb{R} \times V\right)$. We rewrite (1) as

$$
\partial_{t} f_{\varepsilon}+v \partial_{x} g_{\varepsilon}=\frac{1}{\varepsilon^{2}}\left(\rho_{\varepsilon}-f_{\varepsilon}\right)-\frac{v}{\varepsilon} \partial_{x} \rho_{\varepsilon}
$$

which motivates the following two step splitting scheme:

Given a uniform subdivision of step $\Delta t$ of $[0, \infty)$ and knowing $f^{n}$, which is expected to approximate $f^{\varepsilon}(n \Delta t, x, v), n \in \mathbb{N}$

Step 1.- Solve on the time interval $[n \Delta t,(n+1) \Delta t)$ the stiff ODE

$$
\partial_{t} f=\frac{1}{\varepsilon^{2}}(\rho-f)-\frac{1}{\varepsilon} v \partial_{x} \rho
$$

Since the average over $V$ of the right hand side vanishes, the macroscopic density is not modified during this time step, that is,

$$
\rho^{n+1 / 2}=\int_{V} f^{n+1} \mathrm{~d} \mu(v)=\rho^{n} .
$$

Moreover, (11) also defines the evolution of the fluctuation

$$
\partial_{t} g=-\frac{1}{\varepsilon^{2}} g-\frac{1}{\varepsilon^{2}} v \partial_{x} \rho
$$

Step 2.- Solve on the time interval $[n \Delta t,(n+1) \Delta t)$ :

$$
\partial_{t} f+v \partial_{x} g=0 \quad \text { and } \quad \partial_{t} g=0 .
$$

This defines, $f^{n+1}$ and

$$
\rho^{n+1}=\int_{V} f^{n+1} \mathrm{~d} \mu(v)
$$

We emphasize that the index $\varepsilon$ has been dropped for notational convenience. Note that, in the second step, the convective term involves a characteristic speed of order $\mathcal{O}(1)$ only and that we will not force any update on $g$ as $g^{n+1 / 2}=\left(f^{n+1 / 2}-\rho^{n+1 / 2}\right) / \varepsilon$. This update might make the relation between $f$ and $g$ consistent at the end of the second step but it leads to undesirable numerical divisions by the small parameter $\varepsilon$; but, for well-prepared initial data, this consistency can be imposed at the beginning. Similar arguments were already given in $[30,31]$ to avoid this update of the fluctuations $g$. 


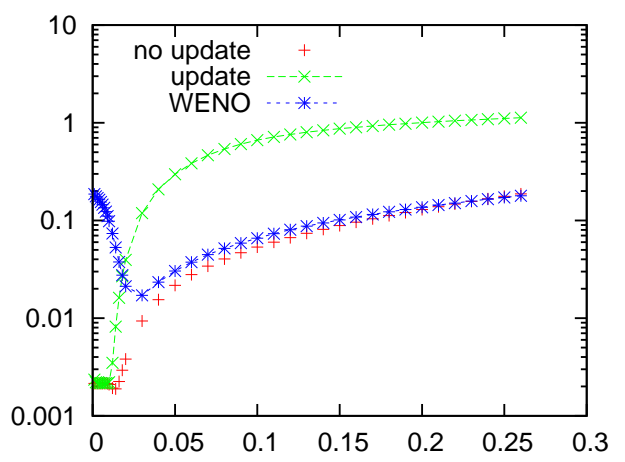

FIGURE 1. $L_{t, x, v^{-}}^{2}$ error of the distribution function $f$ with respect to the solution of the heat equation with a symmetric initial data as in Section 5 with a mesh of 100x100 with respect to $\varepsilon$.

Before proceeding further with the analysis of this kinetic method, we show in Figure $1 \mathrm{a}$ comparison between the results of the three discussed kinetic methods: a semi-lagrangian PWENO6,4-interpolation scheme [10] (SL-WENO), the asymptotic preserving method without update of $g$ proposed above and the asymptotic preserving method with update of $g$. In all cases, we show the $L_{t, x, v}^{2}$-error between the kinetic results and the solutions of the heat equation, its $\varepsilon \rightarrow 0$ asymptotic limit, in a log-plot depending on $\varepsilon$. The results show that the kinetic scheme proposed in this paper works perfectly in the $\varepsilon \rightarrow 0$ regime while both the updated scheme and the SLWENO scheme do not describe well the asymptotic limit. It is important to point out that all the schemes are computed with the parabolic CFL condition corresponding to the limiting heat equation. We also note that for larger values of $\varepsilon$ the difference between the SL-WENO method and the asymptotic preserving scheme becomes small which shows the ability of the proposed scheme to capture the behavior of the kinetic equation, for moderately small value of the mean free path as well, with a considerable gain of CPU time. It is also worthy to emphasize that the results are given for a fixed mesh 100x100, so that as $\varepsilon \rightarrow 0$, the SL-WENO method cannot work, the velocities being of order $\mathcal{O}(1 / \varepsilon)$. In order to get accurate results comparable to those obtained with the asymptotic-induced scheme, the SL-WENO method would require larger and larger meshes in velocity and smaller and smaller time steps as $\varepsilon \rightarrow 0$. This leads to an unbearable computational cost for such a simple equation. 
We can simplify the first step by keeping only the leading contribution in $\varepsilon$ and, by explicitly solving Equations (11) and (12) that define $f^{n+1 / 2}$, $\rho^{n+1 / 2}$ and $g^{n+1 / 2}$, leading to

$$
g^{n+1 / 2}=e^{-\Delta t / \varepsilon^{2}} g^{n}-\left(1-e^{-\Delta t / \varepsilon^{2}}\right) v \partial_{x} \rho^{n}
$$

and

$$
f^{n+1 / 2}=e^{-\Delta t / \varepsilon^{2}} f^{n}+\left(1-e^{-\Delta t / \varepsilon^{2}}\right) \rho^{n},
$$

keeping in mind that $\rho^{n+1 / 2}=\rho^{n}=\int_{V} f^{n} \mathrm{~d} \mu(v)$. The final semi-discrete scheme is summarized as:

Step 1.- Compute

$$
\left\{\begin{array}{l}
g^{n+1 / 2}=e^{-\Delta t / \varepsilon^{2}} g^{n}-\left(1-e^{-\Delta t / \varepsilon^{2}}\right) v \partial_{x} \rho^{n}, \\
f^{n+1 / 2}=e^{-\Delta t / \varepsilon^{2}} f^{n}+\left(1-e^{-\Delta t / \varepsilon^{2}}\right) \rho^{n} .
\end{array}\right.
$$

Remember that $\rho^{n+1 / 2}=\rho^{n}$.

Step 2.- Solve for time $\Delta t$ the convection equation:

$$
\partial_{t} f+v \partial_{x} g=0
$$

to compute the values of $f^{n+1}$ and $\rho^{n+1}$ while $g^{n+1}=g^{n+1 / 2}$.

Remark 2 (Asymptotic Preserving). It is worthwhile mentioning that the scheme is "asymptotic preserving": using (15) and (14) for the completely relaxed model, i.e., $\varepsilon=0$, yields

$$
f^{n+1 / 2}=\rho^{n+1 / 2}, \quad g^{n+1 / 2}=-v \partial_{x} \rho^{n}=-v \partial_{x} \rho^{n+1 / 2},
$$

which coincides with the first order term in the Hilbert expansion. Thus, the first step becomes

$$
\partial_{t} f-v^{2} \partial_{x x}^{2} \rho=0
$$

Integrating over the mesh of velocities leads to the expected heat equation, up to a suitable $v$-mesh definition in order to guarantee $\int_{V} v^{2} \mathrm{~d} v=d$.

Remark 3 (Spatial Derivatives Discretization). One has to take care of the treatment of the space derivative: if one uses the same upwind discretization for evaluating both $-v \partial_{x} \rho$ in the first step and $-v \partial_{x} g$ in the second one, it leads to an unstable scheme for the heat equation. The usual 3-point scheme is obtained by choosing opposite upwind discretization in the successive time steps. Accordingly, for $\varepsilon=0$, the stability of the scheme is guaranteed by the CFL condition $d \Delta t /(\Delta x)^{2} \leq 1 / 2$.

Remark 4 (Stability). The stability condition for the scheme used with $\varepsilon>0$ is less clear, even if a CFL condition close to the parabolic one can be reasonably expected. We refer to [32] for a discussion on a semiimplicit version of the proposed scheme. This difficulty has motivated the development of implicit methods, as in [24, 25]. 
Remark 5 (Current and Distribution Computation). Due precisely to the separation between fluctuations and relaxation towards the homogeneous density we impose in the scheme and taking into account the comments above regarding asymptotic preservation, we need to compute and reconstruct $J$ and $f$ to compare to other methods. In fact, since currents appear due to fluctuations, it is intuitive to reconstruct it as

$$
J^{n+1}=\int_{V} v g^{n+1} \mathrm{~d} \mu(v) .
$$

Due to the Hilbert expansion approach, we will consider the reconstructed distribution given by $\rho^{n+1}+\varepsilon g^{n+1}$.

Let us restrict from now on to the case of the normalized Lebesgue measure $\mathrm{d} \mu(v)$ on the velocity space $[-1,1]$. The space interval $\left[X_{\min }, X_{\max }\right]$ is uniformly discretized in $N_{x}-1$ intervals with points $x_{i}=i \Delta x$ from $i=0, \ldots, N_{x}-1$ and the velocity interval $[-1,1]$ is discretized analogously in $N_{v}-1$ intervals with points $v_{j}=j \Delta v$ from $i=0, \ldots, N_{v}-1$. For further purposes, it is convenient to introduce the sets

$$
\begin{aligned}
& V_{+}=\left\{j \in\left\{0, N_{v}-2\right\} \text { such that } v_{j}>0\right\}, \\
& V_{-}=\left\{j \in\left\{0, N_{v}-2\right\} \text { such that } v_{j}<0\right\} .
\end{aligned}
$$

Let us specify our discrete scheme to the case of simple upwind discretization $\mathbb{D}_{j}$ of the spatial differential operator $-v_{j} \partial_{x}$ with $\overline{\mathbb{D}}_{j}$ being its alternate direction: for a given sequence $\left(\varphi_{i}\right)_{i \in \mathbb{N}}$, we set

$$
\begin{aligned}
{\left[\mathbb{D}_{j} \varphi\right]_{i} } & = \begin{cases}-v_{j}\left(\varphi_{i}-\varphi_{i-1}\right) & \text { if } v_{j} \in V_{+}, \\
-v_{j}\left(\varphi_{i+1}-\varphi_{i}\right) & \text { if } v_{j} \in V_{-},\end{cases} \\
{\left[\overline{\mathbb{D}}_{j} \varphi\right]_{i} } & = \begin{cases}-v_{j}\left(\varphi_{i+1}-\varphi_{i}\right) & \text { if } v_{j} \in V_{+}, \\
-v_{j}\left(\varphi_{i}-\varphi_{i-1}\right) & \text { if } v_{j} \in V_{-} .\end{cases}
\end{aligned}
$$

More advanced non-centered non linear distinct numerical fluxes for $-v_{j} \partial_{x}$, such as flux limiting ones, may be chosen. Similarly, we could use a non uniform time mesh. However, this might complicate boundary conditions below to preserve mass and it will certainly change the relaxed asymptotic scheme. The fully discrete scheme summarizes as

Step 1.- Compute

$$
\left\{\begin{array}{l}
g_{i, j}^{n+1 / 2}=e^{-\Delta t / \varepsilon^{2}} g_{i, j}^{n}+\left(1-e^{-\Delta t / \varepsilon^{2}}\right) \overline{\mathbb{D}}_{j} \rho_{i}^{n} \\
f_{i, j}^{n+1 / 2}=e^{-\Delta t / \varepsilon^{2}} f_{i, j}^{n}+\left(1-e^{-\Delta t / \varepsilon^{2}}\right) \rho_{i}^{n}
\end{array},\right.
$$

with

$$
\rho_{i}^{n+1 / 2}=\rho_{i}^{n}=\frac{\Delta v}{2} \sum_{j=0}^{N_{v}-2} f_{i, j}^{n} .
$$


Step 2.- Solve for time $\Delta t$ the convection-like equation:

$$
f_{i, j}^{n+1}=f_{i, j}^{n+1 / 2}+\Delta t \mathbb{D}_{j} g_{i, j}^{n+1 / 2}
$$

to compute the values of $f^{n+1}$ and $\rho^{n+1}$ while $g^{n+1}=g^{n+1 / 2}$.

Remark 6 (Maximum Principle). We point out again that the scheme is specifically designed for the small $\varepsilon$ regime, and there is no guarantee about the accuracy of the results when $\varepsilon$ becomes large. In particular difficulties might arise with the maximum principle. Indeed, in Step 1, given a non negative $f^{n}$, (18) returns a non negative $f^{n+1 / 2}$, but this property is not naturally preserved in Step 2, see (19).

Finally, we need to impose boundary conditions on the advection step ensuring the total mass conservation. With this aim, we need

$$
\sum_{i=1}^{N_{x}-2} \sum_{j=0}^{N_{v}-2} \mathbb{D}_{j} g_{i, j}^{n+1 / 2}=0
$$

which is equivalent, by summing the telescopic series appearing due to the definition of the upwinding operators, to

$$
\sum_{v_{j} \in V_{+}} v_{j}\left(g_{N_{x}-2, j}^{n+1 / 2}-g_{0, j}^{n+1 / 2}\right)+\sum_{v_{j} \in V_{-}} v_{j}\left(g_{N_{x}-1, j}^{n+1 / 2}-g_{1, j}^{n+1 / 2}\right)=0 .
$$

¿From this, we will impose as boundary conditions for the fluctuations:

$$
g_{0, k}^{n+1 / 2}=\frac{-1}{v_{k} \#\left[V_{+}\right]} \sum_{v_{j} \in V_{-}} v_{j} g_{1, j}^{n+1 / 2}
$$

for $k \in V_{+}$and

$$
g_{N_{x}-1, k}^{n+1 / 2}=\frac{-1}{v_{k} \#\left[V_{-}\right]} \sum_{v_{j} \in V_{+}} v_{j} g_{N_{x}-2, j}^{n+1 / 2}
$$

for $k \in V_{-}$, where $\#[B]$ is the cardinal of the set $B$. Let us remark that the previous boundary condition in the complete relaxed scheme, $\varepsilon \rightarrow 0$, coincides with the Neumann boundary condition for the density, i.e.,

$$
\rho_{0}^{n}=\rho_{1}^{n} \quad \text { and } \quad \rho_{N_{x}-1}^{n}=\rho_{N_{x}-2}^{n} .
$$

The scheme described above gives a simple way to compute the solution of (1)-(2), and the associated macroscopic density, that has to be compared, both in terms of accuracy and computational cost, to the direct evaluation, see Fig. 1, and computation of the solution of the heat equation (4) and the different approximations by the closure strategies.

The method adapts easily to more complicated models: gas dynamics $[30,31]$, radiative transfer [21, 28], fluid-particles flows [9]. It can be also 
incorporated in a domain decomposition method to deal with space varying mean free path, in the spirit of $[23,49]$.

\section{Numerical Schemes for Closure Approximations}

Next, the idea to treat the hyperbolic system (9) or the conservation equation (8) is two-fold:

(1) We introduce additional unknowns and parameters and the equations are seen as the relaxation limit of an extended system, in the spirit of general methods described in [41],

(2) The relaxation system is interpreted itself as a kinetic equation with a discrete set of velocities to which we apply the splitting algorithm described above.

4.1. Relaxation Method for the First-Order Closure. We will at first focus on developing a numerical scheme for the first order closure (9). The nonlinear system (9) can be seen as the limit, as $\alpha$ tends to 0 , of

$$
\begin{aligned}
& \partial_{t} \rho+\partial_{x} J=0, \\
& \varepsilon^{2} \partial_{t} J+\partial_{x} z=-J, \\
& \partial_{t} z+\varepsilon^{2} \lambda^{2} \partial_{x} J=\frac{1}{\alpha}(\rho \psi(\varepsilon J / \rho)-z) .
\end{aligned}
$$

Let us define $u:=\varepsilon J / \rho$. Recall that $u$ should be small of order $\mathcal{O}(\varepsilon)$, see [13]. This system involves an additional unknown $z(t, x)$ and the parameters $\lambda$ (convection speed) and $\alpha$ (relaxation parameter). Actually we relax on the quantity $\varepsilon^{2} J$ so that we consider the velocity in (22) rescaled by $\varepsilon$ (that fits dimensional considerations). The advantage in considering (20)-(22) is that now we have to deal with simple convection equations, the convection part being linear, and all nonlinearities only appear in the (zeroth order) source terms. This idea is reminiscent to the introduction of kinetic schemes in $[4,20,45,39,38]$, and relaxation methods for conservation laws [29]. We refer to $[1,44]$ for further details and references. This approach can be used also to treat degenerate diffusion equations [41].

Let us find the constraints on the additional velocities $\pm \lambda$ that should be large enough to propagate enough information to reconstruct the behavior of (9). It is important to check whether the condition becomes more constrained as $\varepsilon$ tends to 0 . To this end, let us perform the Chapman-Enskog reasoning; we expand (22) with respect to $\alpha$ considering $\varepsilon$ to be small. We have

$z=\rho \psi(u)-\alpha\left(\partial_{t} z+\varepsilon^{2} \lambda^{2} \partial_{x} J\right)=\rho \psi(u)-\alpha\left(\partial_{t}(\rho \psi(u))+\varepsilon^{2} \lambda^{2} \partial_{x} J\right)+\mathcal{O}\left(\alpha^{2}\right)$, 
by (22)-(21). Thus, (21) can be recast as

$$
\varepsilon^{2} \partial_{t} J+\partial_{x}(\rho \psi(u))+J=\alpha \varepsilon^{2} \lambda^{2} \partial_{x x}^{2} J+\alpha \partial_{x t}^{2}(\rho \psi(u))+\mathcal{O}\left(\alpha^{2}\right) .
$$

Let us compute the leading contribution in the last term; by using (20) and (21), we get

$$
\partial_{t}(\rho \psi(u))=-\psi(u) \partial_{x} J+\rho \psi^{\prime}(u) \partial_{t} u
$$

But

$$
\partial_{t}(\rho \psi(u))=\left(u \psi^{\prime}(u)-\psi(u)\right) \partial_{x} J-\frac{\psi^{\prime}(u)}{\varepsilon}\left(J+\partial_{x} z\right) .
$$

Now, considering that formally $J+\partial_{x} z$ is of order $\mathcal{O}(\varepsilon)$ at least, that $\psi$ is an even function and using the approximation $\psi(u)=\psi(0)+\mathcal{O}\left(\varepsilon^{2}\right)$, with $\psi(0)>0$, we get

$$
\partial_{t}(\rho \psi(u))+\psi(0) \partial_{x} J=\mathcal{O}(\varepsilon)
$$

so that

$$
\varepsilon^{2} \partial_{t} J+\partial_{x}(\rho \psi(u))+J=\alpha \partial_{x}\left(\left(\varepsilon^{2} \lambda^{2}-\psi(0)\right) \partial_{x} J\right)+\mathcal{O}\left(\alpha^{2}, \varepsilon\right) .
$$

Consequently, as soon as $\varepsilon|\lambda|>\sqrt{\psi(0)}$, the parabolicity is ensured. It is certainly natural to find that the speeds tend to infinity as $\varepsilon$ tends to 0 since we want to approximate the heat equation. Now, we need to diagonalize System (20)-(22). Since the quantity $\varepsilon \lambda$ remains bounded from below, we denote it by $\mu$. We define

$$
\begin{aligned}
f_{0} & =\mu^{2} \rho-z \\
f_{ \pm} & =\frac{1}{2}(z \pm \varepsilon \mu J) .
\end{aligned}
$$

Of course, we have

$$
z=f_{+}+f_{-} \text {and } J=\frac{f_{+}-f_{-}}{\varepsilon \mu} \text { and } \rho=\frac{f_{0}+f_{+}+f_{-}}{\mu^{2}} .
$$

Noting that

$$
J= \pm \frac{2 f_{ \pm}-z}{\varepsilon \mu},
$$

the new system we are interested in is

$$
\begin{aligned}
\partial_{t} f_{0} & =-\frac{1}{\alpha}(\rho \psi(u)-z) \\
\partial_{t} f_{ \pm} \pm \frac{\mu}{\varepsilon} \partial_{x} f_{ \pm} & =-\frac{f_{ \pm}}{\varepsilon^{2}}+\frac{z}{2 \varepsilon^{2}}+\frac{1}{2 \alpha}(\rho \psi(u)-z) .
\end{aligned}
$$

The system shares some structures with the kinetic equation analyzed in the previous section. This similarity will be used to design a new scheme that will be expressed only in terms of the macroscopic quantities $\rho$ and $J$. Since we have two small parameters, we can use a double splitting method, 
i.e., by splitting with respect to $\varepsilon$ inside the splitting with respect to $\alpha$, that is:

Step 1.- Solve

$$
\begin{aligned}
\partial_{t} f_{0} & =0 \\
\partial_{t} f_{ \pm} \pm \frac{\mu}{\varepsilon} \partial_{x} f_{ \pm} & =-\frac{f_{ \pm}}{\varepsilon^{2}}+\frac{z}{2 \varepsilon^{2}} .
\end{aligned}
$$

This system is again stiff as $\varepsilon$ tends to 0 . Let us solve it with the splitting method described in Section 3: we introduce the intermediate variables

$$
g_{ \pm}:=\frac{2 f_{ \pm}-z}{2 \varepsilon}= \pm \frac{\mu J}{2}
$$

and rewrite (29) as

$$
\partial_{t} f_{ \pm} \pm \mu \partial_{x} g_{ \pm}=-\frac{g_{ \pm}}{\varepsilon} \mp \frac{\mu}{2 \varepsilon} \partial_{x} z
$$

Solve

$$
\begin{aligned}
& \text { Step 1.1.- } \quad \partial_{t} f_{ \pm}=-\frac{f_{ \pm}}{\varepsilon^{2}}+\frac{z}{2 \varepsilon^{2}} \mp \frac{\mu}{2 \varepsilon} \partial_{x} z, \\
& \partial_{t} g_{ \pm}=-\frac{g_{ \pm}}{\varepsilon^{2}} \mp \frac{\mu}{2 \varepsilon^{2}} \partial_{x} z
\end{aligned}
$$

where the initial condition for the ODEs are the values computed in the previous step and solve

$$
\begin{array}{ll}
\text { Step 1.2.- } & \partial_{t} f_{ \pm} \pm \mu \partial_{x} g_{ \pm}=0, \\
& \partial_{t} g_{ \pm}=0,
\end{array}
$$

where the initial conditions are, for $f_{ \pm}$, the ones obtained by Step 1.1. For $g_{ \pm}$, we update them in terms of the flux $g_{ \pm}(0)= \pm \mu J / 2= \pm\left(f_{+}-\right.$ $\left.f_{-}\right) / 2 \varepsilon$. Note that here the update is necessary since the goal of the scheme is actually to compute the macroscopic flux and the microscopic quantities $f_{ \pm}, f_{0}$ and $g_{ \pm}$are only auxiliary devices.

Note that, during Step 1.1, $\partial_{t} z=0$. Let us now specify our fully discrete kinetic scheme. As in the previous section, let us choose $\mathbb{D}_{ \pm}$an upwind discretization of the spatial differential operator $\mp \mu \partial_{x}$ and $\overline{\mathbb{D}}_{ \pm}$its alternate direction version, see (3). The fully discrete kinetic scheme in this step reads as 


\section{Step 1.1.-(Micro)}

$$
\begin{aligned}
& g_{ \pm}^{n+1 / 4}=e^{-\Delta t / \varepsilon^{2}} g_{ \pm}^{n}+\left(1-e^{-\Delta t / \varepsilon^{2}}\right) \frac{1}{2} \overline{\mathbb{D}}_{ \pm}\left(f_{+}^{n}+f_{-}^{n}\right), \\
& f_{ \pm}^{n+1 / 4}=e^{-\Delta t / \varepsilon^{2}} f_{ \pm}^{n}+\left(1-e^{-\Delta t / \varepsilon^{2}}\right)\left(\frac{f_{+}^{n}+f_{-}^{n}+\varepsilon \overline{\mathbb{D}}_{ \pm}\left(f_{+}^{n}+f_{-}^{n}\right)}{2}\right), \\
& f_{0}^{n+1 / 4}=f_{0}^{n} .
\end{aligned}
$$

At the microscopic level, after Step 1.1, $g_{+}^{n+1 / 4} \neq-g_{-}^{n+1 / 4}$.

\section{Step 1.2.-(Micro)}

$$
\begin{aligned}
g_{ \pm}^{n+1 / 2} & = \pm \frac{f_{+}^{n+1 / 4}-f_{-}^{n+1 / 4}}{2 \varepsilon}, \\
f_{ \pm}^{n+1 / 2} & =f_{ \pm}^{n+1 / 4}+\Delta t \mathbb{D}_{ \pm}\left(g_{ \pm}^{n+1 / 4}\right) \\
f_{0}^{n+1 / 2} & =f_{0}^{n+1 / 4}, \\
(\rho \psi)^{n+1 / 2}= & \frac{1}{\mu^{2}}\left(f_{+}^{n+1 / 2}+f_{-}^{n+1 / 2}+f_{0}^{n+1 / 2}\right) \\
& \quad \times \psi\left(\frac{\mu\left(f_{+}^{n+1 / 2}-f_{-}^{n+1 / 2}\right)}{f_{+}^{n+1 / 2}+f_{-}^{n+1 / 2}+f_{0}^{n+1 / 2}}\right)
\end{aligned}
$$

Note that, after Step 1.2, we have $g_{+}^{n+1 / 2}=-g_{-}^{n+1 / 2}$, as it is the case in the continuous setting. That is the reason why the macroscopic quantities can only be expressed at the end of Step 1 as a whole, and not at the end of Step 1.1. The macroscopic scheme summarizes in this step as:

\section{Step 1.-(Macro)}

$$
\begin{aligned}
z^{n+1 / 2}=z^{n}+ & \frac{\varepsilon\left(1-e^{-\Delta t / \varepsilon^{2}}\right)}{2}\left(\overline{\mathbb{D}}_{+}\left(z^{n}\right)+\overline{\mathbb{D}}_{-}\left(z^{n}\right)\right) \\
+\Delta & {\left[\mathbb{D}_{+}\left(e^{-\Delta t / \varepsilon^{2}} \frac{\mu J^{n}}{2}+\left(1-e^{-\Delta t / \varepsilon^{2}}\right) \frac{\overline{\mathbb{D}}_{+}\left(z^{n}\right)}{2}\right)\right.} \\
& \left.+\mathbb{D}_{-}\left(e^{-\Delta t / \varepsilon^{2}} \frac{\left(-\mu J^{n}\right)}{2}+\left(1-e^{-\Delta t / \varepsilon^{2}}\right) \frac{\overline{\mathbb{D}}_{-}\left(z^{n}\right)}{2}\right)\right],
\end{aligned}
$$




$$
\begin{aligned}
& J^{n+1 / 2}=e^{-\Delta t / \varepsilon^{2}} J^{n}+\frac{1-e^{-\Delta t / \varepsilon^{2}}}{2 \mu}\left(\overline{\mathbb{D}}_{+}\left(z^{n}\right)-\overline{\mathbb{D}}_{-}\left(z^{n}\right)\right) \\
& +\frac{\Delta t}{\varepsilon \mu}\left[\mathbb{D}_{+}\left(e^{-\Delta t / \varepsilon^{2}} \frac{\mu J^{n}}{2}+\left(1-e^{-\Delta t / \varepsilon^{2}}\right) \frac{\overline{\mathbb{D}}_{+}\left(z^{n}\right)}{2}\right)\right. \\
& \left.-\mathbb{D}_{-}\left(e^{-\Delta t / \varepsilon^{2}} \frac{\left(-\mu J^{n}\right)}{2}+\left(1-e^{-\Delta t / \varepsilon^{2}}\right) \frac{\overline{\mathbb{D}}_{-}\left(z^{n}\right)}{2}\right)\right], \\
& \rho^{n+1 / 2}=\rho^{n}+\frac{\Delta t}{\mu^{2}}\left(\mathbb{D}_{+}\left(e^{-\Delta t / \varepsilon^{2}} \frac{\mu J^{n}}{2}+\left(1-e^{-\Delta t / \varepsilon^{2}}\right) \frac{\overline{\mathbb{D}}_{+}\left(z^{n}\right)}{2}\right)\right. \\
& \left.+\mathbb{D}_{-}\left(e^{-\Delta t / \varepsilon^{2}} \frac{\left(-\mu J^{n}\right)}{2}+\left(1-e^{-\Delta t / \varepsilon^{2}}\right) \frac{\overline{\mathbb{D}}_{-}\left(z^{n}\right)}{2}\right)\right) \text {. }
\end{aligned}
$$

Let us remark that the second term in $z^{n+1 / 2}$ is of order $\varepsilon$ and thus, it will be omitted in the computations below. Now, we can write the relaxation step with respect to the parameter $\alpha$ :

Step 2.- Solve the ODE

$$
\begin{aligned}
\partial_{t} f_{0} & =-\frac{1}{\alpha}(\rho \psi(u)-z), \\
\partial_{t} f_{ \pm} & =\frac{1}{2 \alpha}(\rho \psi(u)-z),
\end{aligned}
$$

that is, since $\partial_{t} J=0$ and $z=2 f_{ \pm} \mp \varepsilon \mu J$ by virtue of (25),

$$
\begin{aligned}
\partial_{t} f_{0} & =-\frac{1}{\alpha}(\rho \psi(u)-z), \\
\partial_{t} f_{ \pm} & =-\frac{1}{\alpha} f_{ \pm}+\frac{1}{2 \alpha}(\rho \psi(u) \pm \varepsilon \mu J),
\end{aligned}
$$

with as initial conditions the values computed from Step 1. In this last step, we also note that $\partial_{t} \rho=0$, see (24) and (25), and that, consequently, $u$ is constant. The fully discrete kinetic scheme summarizes in this step as

\section{Step 2.-(Micro)}

$$
\begin{aligned}
& f_{ \pm}^{n+1}=e^{-\Delta t / \alpha} f_{ \pm}^{n+1 / 2}+\frac{1}{2}\left(1-e^{-\Delta t / \alpha}\right)\left((\rho \psi)^{n+1 / 2}+2 \varepsilon g_{ \pm}^{n+1 / 2}\right) \\
& f_{0}^{n+1}=f_{0}^{n}+\left(1-e^{-\Delta t / \alpha}\right)\left(f_{+}^{n}+f_{-}^{n}-(\rho \psi)^{n+1 / 2}\right)
\end{aligned}
$$

In this last step, we do not update $g$, since $\partial_{t} J=0$. Denoting by $\psi^{n+1 / 2}$ the quantity $\psi\left(\varepsilon J^{n+1 / 2} / \rho^{n+1 / 2}\right)$, we deduce the following macroscopic scheme for the second step: 


\section{Step 2.-(Macro)}

$$
\begin{aligned}
z^{n+1} & =e^{-\Delta t / \alpha} z^{n+1 / 2}+\left(1-e^{-\Delta t / \alpha}\right) \rho^{n+1 / 2} \psi^{n+1 / 2}, \\
J^{n+1} & =J^{n+1 / 2}, \\
\rho^{n+1} & =\rho^{n+1 / 2} .
\end{aligned}
$$

Remark 7 (Splitting Order). We choose a semi-linear relaxation method to have to deal only with transport-like equations, that is, move the nonlinearities to the right-hand side, as source terms. So it is natural to take this precise order of splitting, since $\alpha$ must be the first one to tend to 0 , so that we can keep a non-zero $\varepsilon$, even if it is small.

Remark 8 (Initial Conditions). In order to prevent an initial layer from appearing [42] in the $\alpha$-splitting, we need to prescribe well-prepared initial conditions taking into account both splittings as:

$$
\begin{aligned}
& \rho(0, x)=\rho^{0}(x), \\
& J(0, x)=J^{0}(x), \\
& z(0, x)=\rho^{0}(x) \psi\left(\varepsilon J^{0}(x) / \rho^{0}(x)\right)=: z^{0}(x),
\end{aligned}
$$

corresponding to the choice of the equilibrium state for the (hyperbolic) $\alpha$ splitting, as in the classical relaxation approach.

Remark 9 (Boundary Conditions). We consider, for a computation domain $\left[X_{\text {min }}, X_{\text {max }}\right]$, Neumann conditions for $\rho$ and $z$,

$$
\rho_{0}^{n}=\rho_{1}^{n} \quad \rho_{N_{x}-1}^{n}=\rho_{N_{x}-2}^{n} \quad \text { and } \quad z_{0}^{n}=z_{1}^{n} \quad z_{N_{x}-1}^{n}=z_{N_{x}-2}^{n} .
$$

and

$$
J_{0}^{n}=-J_{1}^{n} \quad J_{N_{x}-1}^{n}=-J_{N_{x}-2}^{n} .
$$

These conditions guarantee the conservation of the total mass.

Let us now have a look at the limits as $\alpha$ tends to 0 :

$$
\begin{aligned}
J^{n+1}=e^{-\Delta t / \varepsilon^{2}} J^{n} & +\frac{1-e^{-\Delta t / \varepsilon^{2}}}{2 \mu}\left(\overline{\mathbb{D}}_{+}\left(\rho^{n} \psi^{n}\right)-\overline{\mathbb{D}}_{-}\left(\rho^{n} \psi^{n}\right)\right) \\
+ & \frac{\Delta t}{\varepsilon \mu}\left[\mathbb{D}_{+}\left(e^{-\Delta t / \varepsilon^{2}} \frac{\mu J^{n}}{2}+\left(1-e^{-\Delta t / \varepsilon^{2}}\right) \frac{\overline{\mathbb{D}}_{+}\left(\rho^{n} \psi^{n}\right)}{2}\right)\right. \\
& \left.-\mathbb{D}_{-}\left(e^{-\Delta t / \varepsilon^{2}} \frac{\left(-\mu J^{n}\right)}{2}+\left(1-e^{-\Delta t / \varepsilon^{2}}\right) \frac{\overline{\mathbb{D}}_{-}\left(\rho^{n} \psi^{n}\right)}{2}\right)\right], \\
\rho^{n+1}=\rho^{n}+\frac{\Delta t}{\mu^{2}} & \left(\mathbb{D}_{+}\left(e^{-\Delta t / \varepsilon^{2}} \frac{\mu J^{n}}{2}+\left(1-e^{-\Delta t / \varepsilon^{2}}\right) \frac{\overline{\mathbb{D}}_{+}\left(\rho^{n} \psi^{n}\right)}{2}\right)\right. \\
& \left.+\mathbb{D}_{-}\left(e^{-\Delta t / \varepsilon^{2}} \frac{\left(-\mu J^{n}\right)}{2}+\left(1-e^{-\Delta t / \varepsilon^{2}}\right) \frac{\overline{\mathbb{D}}_{-}\left(\rho^{n} \psi^{n}\right)}{2}\right)\right) .
\end{aligned}
$$


We thus obtain a pure transport-projection scheme [1]. Note that, since $\Delta t e^{-\Delta t / \varepsilon^{2}}=\mathcal{O}\left(\varepsilon^{2}\right)$ and

$$
\left(\mathbb{D}_{+}\left(\frac{\overline{\mathbb{D}}_{+}\left(\rho^{n} \psi(0)\right)}{2}\right)-\mathbb{D}_{-}\left(\frac{\overline{\mathbb{D}}_{-}\left(\rho^{n} \psi(0)\right)}{2}\right)\right)=\mathcal{O}(\varepsilon),
$$

the scheme is still reasonable for small $\varepsilon$. The convergence in $\alpha$ of the schemes has been checked numerically. In Figure 2, we show the $L_{t, x^{-}}^{2}$ error in densities of the macroscopic method for $\alpha>0$ with respect to the completely relaxed method above $\alpha=0$ for a fixed valued of $\varepsilon=0.01$.

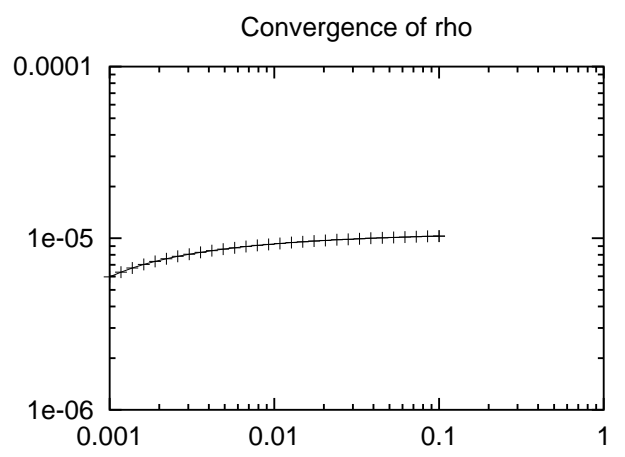

FiguRe 2. $L_{t, x}^{2}$-error of the densities $\rho$ for the $\alpha>0$ method with respect to the completely relaxed scheme $\alpha=0$ for $\varepsilon=0.01$.

Remark 10 (Well-Balanced Scheme). Note that the obtained scheme is well-balanced which means that the stationary states are preserved, if we choose linear discretizations $\mathbb{D}$ : if we take some initial conditions $\rho^{0}$ and $J^{0}$ that satisfy

$$
\begin{gathered}
\partial_{x} J^{0}=0, \\
\partial_{x}\left(\rho^{0} \psi\left(\varepsilon J^{0} / \rho^{0}\right)\right)=-J^{0},
\end{gathered}
$$

so that, in particular, $\partial_{x x}^{2}\left(\rho^{0} \psi\left(\varepsilon J^{0} / \rho^{0}\right)=0\right.$, a direct induction implies that the discrete solution $\left(\rho^{n}, J^{n}\right)_{n}$ is stationary, since $\mathbb{D}_{ \pm}\left(J^{0}\right)=0=\ldots=$ $\mathbb{D}_{ \pm}\left(J^{n}\right)$ and $\mathbb{D}_{ \pm}\left(\overline{\mathbb{D}}_{ \pm}\left(\rho^{0} \psi\left(\varepsilon J^{0} / \rho^{0}\right)\right)=0=\ldots=\mathbb{D}_{ \pm}\left(\overline{\mathbb{D}}_{ \pm}\left(\rho^{n} \psi\left(\varepsilon J^{n} / \rho^{n}\right)\right)\right.\right.$, for all $n \in \mathbb{N}$; see (36).

In turn, the limit $\varepsilon \rightarrow 0$ gives

$$
\rho^{n+1}=\rho^{n}+\frac{\Delta t}{\mu^{2}}\left(\mathbb{D}_{+}\left(\frac{\overline{\mathbb{D}}_{+}\left(\rho^{n} \psi(0)\right)}{2}\right)+\mathbb{D}_{-}\left(\frac{\overline{\mathbb{D}}_{-}\left(\rho^{n} \psi(0)\right)}{2}\right)\right) .
$$


Let us detail the upwind case: since we have, for any sequence $\left(v_{j}\right)_{j \in \mathbb{Z}}$ and for $j \in \mathbb{Z}$,

$$
\begin{aligned}
\mathbb{D}_{+}\left(\overline{\mathbb{D}}_{+}(v)\right)_{j} & =\frac{-\mu}{\Delta x}\left(\left(\frac{-\mu}{\Delta x}\left(v_{k+1}-v_{k}\right)_{k}\right)_{j}-\left(\frac{-\mu}{\Delta x}\left(v_{k+1}-v_{k}\right)_{k}\right)_{j-1}\right), \\
& =\frac{\mu^{2}}{(\Delta x)^{2}}\left(v_{j+1}-2 v_{j}+v_{j-1}\right), \\
\mathbb{D}_{-}\left(\overline{\mathbb{D}}_{-}(v)\right)_{j} & =\mathbb{D}_{+}\left(\overline{\mathbb{D}}_{+}(v)\right)_{j},
\end{aligned}
$$

we get the standard classical 3-point finite difference scheme for the heat equation with conduction $\psi(0)$ :

$$
\rho^{n+1}=\rho^{n}+\psi(0) \frac{\Delta t}{(\Delta x)^{2}}\left(\rho_{j+1}^{n}-2 \rho_{j}^{n}+\rho_{j-1}^{n}\right) .
$$

Remark 11 (Comparison to existing literature). We point out that the strategy differs from the one used in $[5,7]$ where the adopted method, based on well-balanced schemes as introduced in [24, 25], is implicit (see also [16]). The main advantage in the latter is the control on the stability condition. Note however that our method works under the parabolic CFL condition. This could be seen as too restrictive when the kinetic equation or the reduced model is coupled to hydrodynamics, like in applications in radiative transfer $[6,7,16,21]$, but, it is possible in such a context to appeal to a subcycling method where several "parabolic" time steps are performed within a "hyperbolic" time step, see [28].

Besides, the scope of this scheme differs from that of the method described in [41] in the sense that we are interested in computations for a positive value of the parameter $\varepsilon$, not only for the fully relaxed situation.

4.2. Relaxation Method for the Zeroth-Order Closure. Here, we use again analogous ideas to propose a relaxation numerical scheme to solve the zeroth-order closure in (8). The nonlinear equation (8) can be seen as the limit, as $\alpha$ tends to 0 , of

$$
\begin{aligned}
\partial_{t} \rho+\partial_{x} J & =0 \\
\partial_{t} J+\frac{\mu^{2}}{\varepsilon^{2}} \partial_{x} \rho & =-\frac{1}{\alpha}\left[J+\frac{\rho}{\varepsilon} \mathbb{G}\left(\varepsilon \frac{\partial_{x} \rho}{\rho}\right)\right] .
\end{aligned}
$$

Defining now

we have

$$
f_{ \pm}=\frac{\rho}{2} \pm \frac{\varepsilon J}{2 \mu}
$$

$$
\rho=f_{+}+f_{-} \text {and } J=\frac{\mu}{\varepsilon}\left(f_{+}-f_{-}\right) .
$$


The new system we are interested in is

$$
\partial_{t} f_{ \pm} \pm \frac{\mu}{\varepsilon} \partial_{x} f_{ \pm}=\frac{1}{\alpha}\left[\frac{\rho}{2}-f_{ \pm} \mp \frac{\rho}{2 \mu} \mathbb{G}\left(\varepsilon \frac{\partial_{x} \rho}{\rho}\right)\right]
$$

The relaxation scheme follows the same ideas as above. We define the fluctuations as

and then, the equation rewrites as

$$
g_{ \pm}=\frac{1}{\varepsilon} f_{ \pm}-\frac{1}{2 \varepsilon} \rho
$$

$$
\partial_{t} f_{ \pm} \pm \mu \partial_{x} g_{ \pm}=\frac{1}{\alpha}\left[\frac{\rho}{2}-f_{ \pm} \mp \frac{\rho}{2 \mu} \mathbb{G}\left(\varepsilon \frac{\partial_{x} \rho}{\rho}\right)\right] \mp \frac{\mu}{2 \varepsilon} \partial_{x} \rho .
$$

The steps of the method are:

Step 1.- Solve the ODE

$$
\partial_{t} f_{ \pm}=\frac{1}{\alpha}\left[\frac{\rho}{2}-f_{ \pm} \mp \frac{\rho}{2 \mu} \mathbb{G}\left(\varepsilon \frac{\partial_{x} \rho}{\rho}\right)\right] \mp \frac{\mu}{2 \varepsilon} \partial_{x} \rho .
$$

Step 2.- Solve the transport equation

$$
\begin{aligned}
\partial_{t} f_{ \pm} \pm \frac{\mu}{\varepsilon} \partial_{x} f_{ \pm} & =0, \\
\partial_{t} g_{ \pm} & =0 .
\end{aligned}
$$

Here the initial value for the fluctuations for the second step are computed from the values of the first step by:

$$
g_{ \pm}(0)=\frac{1}{\varepsilon} f_{ \pm}-\frac{1}{2 \varepsilon} \rho
$$

The first step of the scheme results into the fully discrete scheme $f_{ \pm}^{n+1 / 2}=$

$=e^{-\Delta t / \alpha} f_{ \pm}^{n}+\frac{\rho^{n}}{2}\left(1-e^{-\Delta t / \alpha}\right)\left[1 \mp \frac{1}{\mu} \mathbb{G}\left(\mp \frac{\varepsilon}{\mu} \frac{\overline{\mathbb{D}}_{ \pm} \rho^{n}}{\rho^{n}}\right)\right]+\alpha\left(1-e^{-\Delta t / \alpha}\right) \frac{1}{2 \varepsilon} \overline{\mathbb{D}}_{ \pm} \rho^{n}$.

The completely relaxed scheme, $\alpha \rightarrow 0$ is

$$
f_{ \pm}^{n+1 / 2}=\frac{\rho^{n}}{2}\left[1+\frac{1}{\mu} \mathbb{G}\left(\frac{\varepsilon}{\mu} \frac{\overline{\mathbb{D}}_{ \pm} \rho^{n}}{\rho^{n}}\right)\right]
$$

where the odd character of $\mathbb{G}$ was used. Taking into account the initialization of the fluctuations above, we get

$$
g_{ \pm}^{n+1 / 2}=\frac{\rho^{n}}{2 \mu \varepsilon} \mathbb{G}\left(\frac{\varepsilon}{\mu} \frac{\overline{\mathbb{D}}_{ \pm} \rho^{n}}{\rho^{n}}\right)
$$

where a term of order $\Delta x^{2}$ was neglected. Now, the values of the solutions in the second step are

$$
f_{ \pm}^{n+1}=f_{ \pm}^{n+1 / 2}+\Delta t \mathbb{D}_{ \pm} g_{ \pm}^{n+1 / 2}
$$


respectively. The use of alternate approximations of the spatial derivatives $\mp \mu \partial_{x}$ is again needed since for all $A \in \mathbb{R}$

$$
\lim _{\varepsilon \rightarrow 0} \frac{\rho}{\varepsilon} \mathbb{G}\left(\varepsilon \frac{A}{\rho}\right)=\frac{1}{3} A .
$$

The complete relaxed scheme in terms of the macroscopic variable $\rho$ reads as

$$
\rho^{n+1}=\rho^{n}+\Delta t\left\{\mathbb{D}_{+}\left[\frac{\rho^{n}}{2 \varepsilon \mu} \mathbb{G}\left(\frac{\varepsilon}{\mu} \frac{\overline{\mathbb{D}}_{+} \rho^{n}}{\rho^{n}}\right)\right]+\mathbb{D}_{-}\left[\frac{\rho^{n}}{2 \varepsilon \mu} \mathbb{G}\left(\frac{\varepsilon}{\mu} \frac{\overline{\mathbb{D}}_{-} \rho^{n}}{\rho^{n}}\right)\right]\right\} .
$$

Due to the diffusive character of the approximation, a parabolic CFL condition for small $\varepsilon$-values, $d \Delta t /(\Delta x)^{2} \leq 1 / 2$, has to be imposed. In this case, following a Chapman-Enskog approach there is no restriction in principle on the value of $\mu>0$ but being of order 1 with respect to $\varepsilon$. The boundary conditions are standard discrete Neumann conditions for $\rho$.

\section{Numerical Results}

5.1. Comparisons between Closures. To start with, we compare the solution of the kinetic equation (1)-(2), computed with the method described in Section 3, and the solutions of the heat equation (4), the zeroth order closure (8), and the first order closure (9); the two last models are evaluated by using the method described in Section 4 . Figure 3 shows the error in a $\log$-log plot with respect to $\varepsilon$, for the symmetric initial data

$$
f_{0}(x, v)= \begin{cases}2 . & \text { for }-0.5 \leq x \leq 0.5 \text { and }-0.5 \leq v \leq 0.5 \\ 1 . & \text { otherwise }\end{cases}
$$

with mesh $N_{x}=N_{v}=100$ and up to time 5 . We used the completely $\alpha$-relaxed version $(\alpha=0)$ of the schemes in Section 4 .

As expected the convergence rates are of order $\mathcal{O}(\varepsilon)$ for all models, confirming the results in [13]. Note however that the macroscopic density $\rho$ is better reproduced by the first order closure and the behavior of the current $J$ is even better captured by this model. For very small values of $\varepsilon$, the density error becomes constant: it is actually dominated by the consistency error, with an error of order $\mathcal{O}\left((\Delta x)^{2}\right)$ (confirmed by changing the mesh size). This is not surprising when thinking of the 3 -point scheme and is due to the splitting method.

Next, we consider a data which is not symmetric with respect to velocity. Figure 4 shows the error in a $\log$-log plot with respect to $\varepsilon$, for the initial 

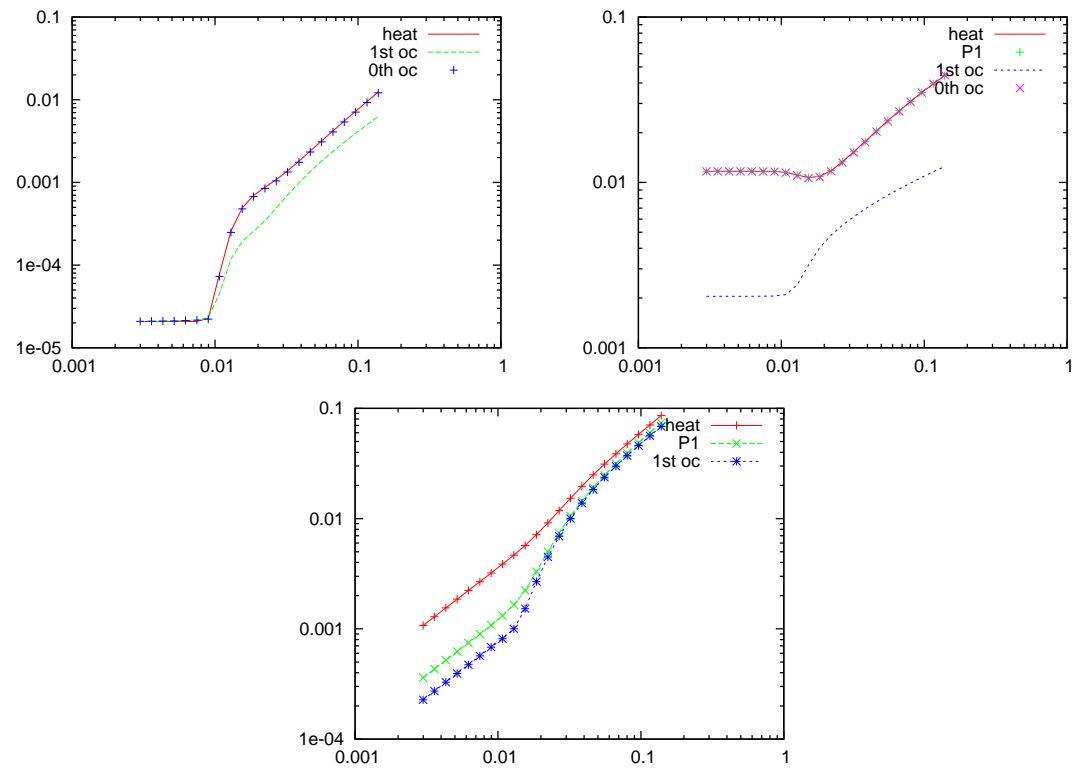

Figure 3. Top left: $L_{t, x}^{2}$ density error, top right: $L_{t, x}^{2}$ current, bottom: $L_{t, x, v}^{2}$ distribution function error between the kinetic result and the corresponding approximations with respect to $\varepsilon$ for the symmetric initial data.

data

$$
f_{0}(x, v)= \begin{cases}2 . & \text { for }-0.5 \leq x \leq 0.5 \text { and }-0.75 \leq v \leq 0.25 \\ 1 . & \text { otherwise }\end{cases}
$$

with mesh $N_{x}=N_{v}=100$ and up to time 5. We still use the completely relaxed framework $\alpha=0$ The previous conclusions are amplified and the advantage of the first order closure appears more strongly, in agreement with conclusions already given in [16].

This is confirmed again by looking at the time evolution of the density and current computed by the different models. Figure 5 corresponds to the evolution of the macroscopic density for the asymmetric initial data with a mesh of $N_{x}=N_{v}=100$ with $\varepsilon=0.1$ and completely relaxed $\alpha=0$ and up to time 5. In Figure 6 we show the corresponding evolution for the first moment $J$. These results favor on the one hand the kinetic and the first order simulation which remain very close, even in this situation where $\varepsilon$ is not particularly small, and on the other hand the zeroth order model which 

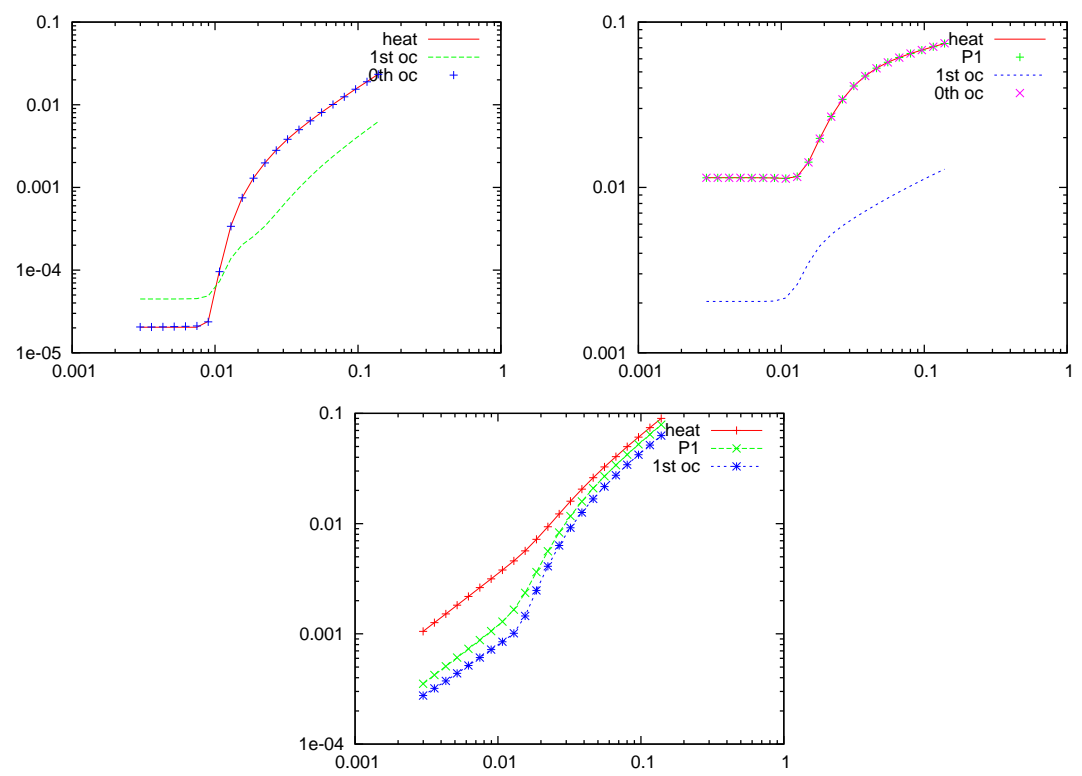

Figure 4. Top left: $L_{t, x}^{2}$ density error, top right: $L_{t, x}^{2}$ current, bottom: $L_{t, x, v}^{2}$ distribution function error between the kinetic result and the corresponding approximations with respect to $\varepsilon$ for the asymmetric initial data.

behaves like the heat equation, far from the profiles obtained by the kinetic computations.

5.2. The Su-Olson Test. This test is a standard benchmark for radiative transfer problems $[43,48,6,7,5]$. Indeed, in radiative transfer the unknown $f$ is the specific intensity of radiations, which interact with the matter through energy exchanges, see e.g. [6, 21]. In this test, the coupling with hydrodynamics is replaced by a simple ODE describing the evolution of the material temperature. More precisely, we have the kinetic equation

$$
\partial_{t} f_{\varepsilon}+\frac{v}{\varepsilon} \partial_{x} f_{\varepsilon}=\frac{1}{\varepsilon^{2}} Q\left(f_{\varepsilon}\right)+\sigma_{a}(\Theta-\rho)+S
$$

coupled with

$$
\partial_{t} \Theta=\sigma_{a}(\rho-\Theta)
$$

where typically in the models, $\Theta=T^{4}$ with $T>0$ the temperature of matter and $S=S(t, x)$ a given source. We propose to solve this stiff coupled problem (45)-(46) with the same approach as in the previous Subsections. 

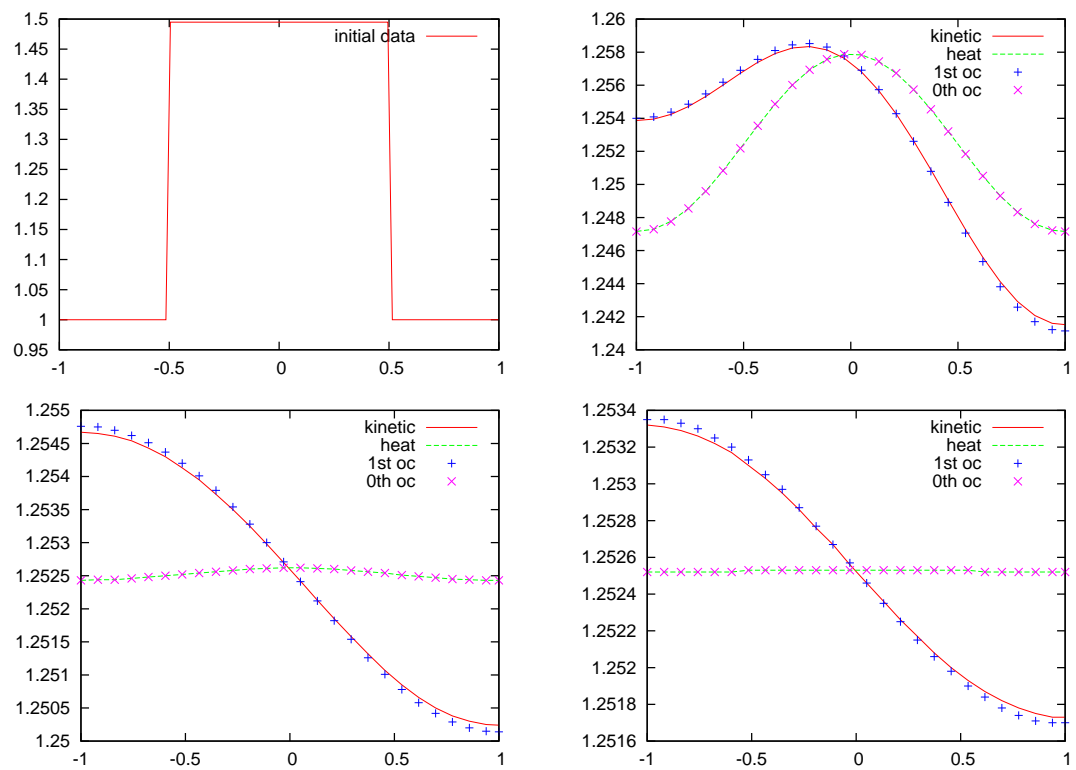

Figure 5. Evolution of the density for the different methods with the asymmetric initial data: top left: initial data, top right: 1.25 time units, bottom left: 2.5 time units, bottom right: 3.75 time units.

We solve the temperature equation at the steps in which the density $\rho$ is constant to have an explicit formula for its solution. We start with the kinetic scheme and we follow the same notation as in Subsection 2.1 skipping some detail. The semi-discrete numerical scheme will summarize as follows:

Step 1.- Compute

$$
\left\{\begin{array}{l}
g^{n+1 / 2}=e^{-\Delta t / \varepsilon^{2}} g^{n}-\left(1-e^{-\Delta t / \varepsilon^{2}}\right) v \partial_{x} \rho^{n}, \\
f^{n+1 / 2}=e^{-\Delta t / \varepsilon^{2}} f^{n}+\left(1-e^{-\Delta t / \varepsilon^{2}}\right) \rho^{n}, \\
\Theta^{n+1 / 2}=e^{-\sigma_{a} \Delta t} \Theta^{n}+\sigma_{a}\left(1-e^{-\sigma_{a} \Delta t}\right) \rho^{n}
\end{array}\right.
$$

Remember that $\rho^{n+1 / 2}=\rho^{n}$.

Step 2.- Solve on a time interval of length $\Delta t$ the convection equation:

$$
\partial_{t} f+v \partial_{x} g=\sigma_{a}(\Theta-\rho)+S
$$



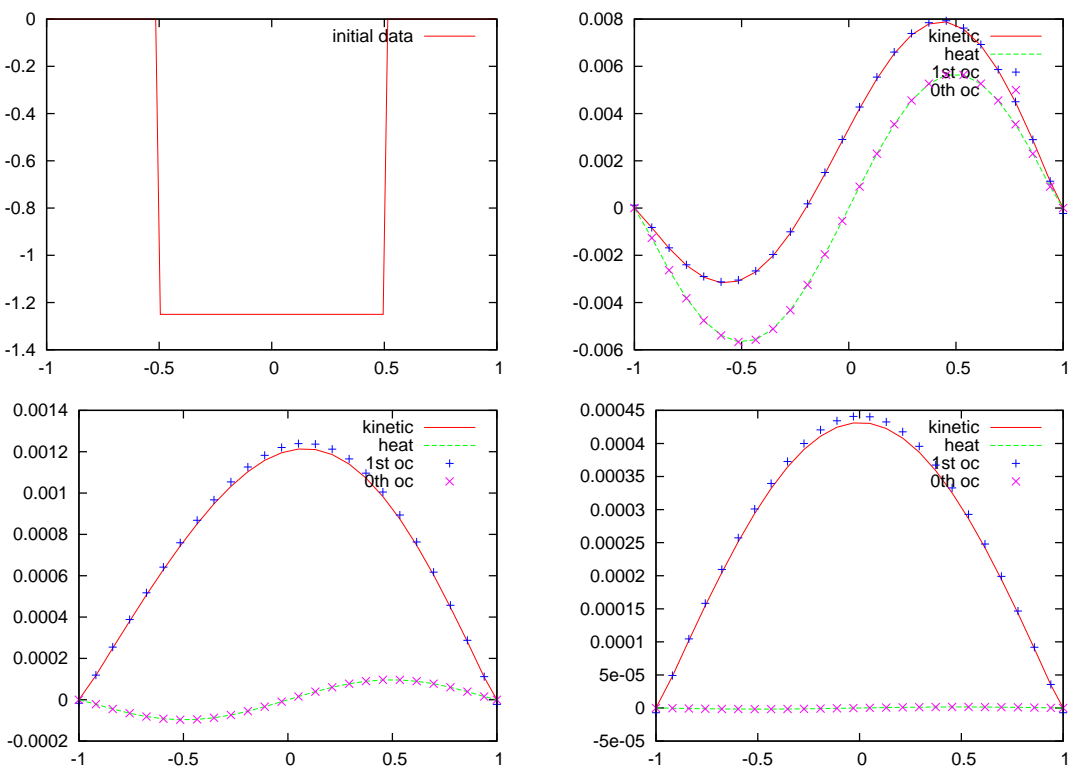

Figure 6 . Evolution of the current for the different methods with the asymmetric initial data: top left: initial data, top right: 1.25 time units, bottom left: 2.5 time units, bottom right: 3.75 time units.

to compute the values of $f^{n+1}$ and $\rho^{n+1}$ while $g^{n+1}=g^{n+1 / 2}$ and $\Theta^{n+1}=\Theta^{n+1 / 2}$. The right-hand side uses the final value provided by Step 1 .

Similar schemes have to be written for the zeroth and first order closures of the Su-Olson test. We start with the first order closure in Subsection 2.2, keeping the notation used therein. The system to solve reads

$$
\left\{\begin{array}{l}
\partial_{t} \varrho+\partial_{x} J=\sigma_{a}(\Theta-\rho)+S, \\
\varepsilon^{2} \partial_{t} J+\partial_{x}(\varrho \psi(\varepsilon J / \varrho))=-J \\
\partial_{t} \Theta=\sigma_{a}(\rho-\Theta) .
\end{array}\right.
$$


The nonlinear system (48) can be seen as the limit, as $\alpha$ tends to 0 , of

$$
\left\{\begin{array}{l}
\partial_{t} \rho+\partial_{x} J=\sigma_{a}(\Theta-\rho)+S \\
\varepsilon^{2} \partial_{t} J+\partial_{x} z=-J \\
\partial_{t} z+\varepsilon^{2} \lambda^{2} \partial_{x} J=\frac{1}{\alpha}(\rho \psi(\varepsilon J / \rho)-z) \\
\partial_{t} \Theta=\sigma_{a}(\rho-\Theta) .
\end{array}\right.
$$

The kinetic scheme will be summarized as

Step 1.- Solve

$$
\begin{aligned}
\partial_{t} f_{0} & =\mu^{2}\left(\sigma_{a}(\Theta-\rho)+S\right), \\
\partial_{t} f_{ \pm} \pm \frac{\mu}{\varepsilon} \partial_{x} f_{ \pm} & =-\frac{f_{ \pm}}{\varepsilon^{2}}+\frac{z}{2 \varepsilon^{2}} \\
\partial_{t} \Theta & =\sigma_{a}(\rho-\Theta),
\end{aligned}
$$

that can be computed as

$$
\begin{aligned}
& \text { Step 1.1.- } \quad \partial_{t} f_{0}=0 \text {, } \\
& \partial_{t} f_{ \pm}=-\frac{f_{ \pm}}{\varepsilon^{2}}+\frac{z}{2 \varepsilon^{2}} \mp \frac{\mu}{2 \varepsilon} \partial_{x} z, \\
& \partial_{t} g_{ \pm}=-\frac{g_{ \pm}}{\varepsilon^{2}} \mp \frac{\mu}{2 \varepsilon^{2}} \partial_{x} z, \\
& \partial_{t} \Theta=\sigma_{a}(\rho-\Theta) \text {, }
\end{aligned}
$$

where the initial condition for the ODEs are the values computed in the previous step and

Step 1.2.-

$$
\begin{aligned}
& \partial_{t} f_{0}=\mu^{2}\left(\sigma_{a}(\Theta-\rho)+S\right) \\
& \partial_{t} f_{ \pm} \pm \mu \partial_{x} g_{ \pm}=0, \\
& \partial_{t} g_{ \pm}=0 \\
& \partial_{t} \Theta=0
\end{aligned}
$$

where the initial conditions are, for $f_{ \pm}$, the ones obtained in Step 1.1. For $g_{ \pm}$, we update them in terms of the flux $g_{ \pm}(0)= \pm \mu J / 2= \pm\left(f_{+}-f_{-}\right) / 2 \varepsilon$.

Step 2.- Solve the ODE

$$
\begin{aligned}
\partial_{t} f_{0} & =-\frac{1}{\alpha}(\rho \psi(u)-z), \\
\partial_{t} f_{ \pm} & =\frac{1}{2 \alpha}(\rho \psi(u)-z), \\
\partial_{t} \Theta & =0 .
\end{aligned}
$$


This kinetic scheme in macroscopic variables is

$$
\begin{aligned}
& z^{n+1 / 2}=z^{n}+\frac{\varepsilon\left(1-e^{-\Delta t / \varepsilon^{2}}\right)}{2}\left(\overline{\mathbb{D}}_{+}\left(z^{n}\right)+\overline{\mathbb{D}}_{-}\left(z^{n}\right)\right) \\
& +\Delta t\left[\mathbb{D}_{+}\left(e^{-\Delta t / \varepsilon^{2}} \frac{\mu J^{n}}{2}+\left(1-e^{-\Delta t / \varepsilon^{2}}\right) \frac{\overline{\mathbb{D}}_{+}\left(z^{n}\right)}{2}\right)\right. \\
& \left.+\mathbb{D}_{-}\left(e^{-\Delta t / \varepsilon^{2}} \frac{\left(-\mu J^{n}\right)}{2}+\left(1-e^{-\Delta t / \varepsilon^{2}}\right) \frac{\overline{\mathbb{D}}_{-}\left(z^{n}\right)}{2}\right)\right], \\
& J^{n+1 / 2}=e^{-\Delta t / \varepsilon^{2}} J^{n}+\frac{1-e^{-\Delta t / \varepsilon^{2}}}{2 \mu}\left(\overline{\mathbb{D}}_{+}\left(z^{n}\right)-\overline{\mathbb{D}}_{-}\left(z^{n}\right)\right) \\
& +\frac{\Delta t}{\varepsilon \mu}\left[\mathbb{D}_{+}\left(e^{-\Delta t / \varepsilon^{2}} \frac{\mu J^{n}}{2}+\left(1-e^{-\Delta t / \varepsilon^{2}}\right) \frac{\overline{\mathbb{D}}_{+}\left(z^{n}\right)}{2}\right)\right. \\
& \left.-\mathbb{D}_{-}\left(e^{-\Delta t / \varepsilon^{2}} \frac{\left(-\mu J^{n}\right)}{2}+\left(1-e^{-\Delta t / \varepsilon^{2}}\right) \frac{\overline{\mathbb{D}}_{-}\left(z^{n}\right)}{2}\right)\right], \\
& \Theta^{n+1 / 2}=e^{-\sigma_{a} \Delta t} \Theta^{n}+\sigma_{a}\left(1-e^{-\sigma_{a} \Delta t}\right) \rho^{n}, \\
& \rho^{n+1 / 2}=\rho^{n}+\frac{\Delta t}{\mu^{2}}\left(\mathbb{D}_{+}\left(e^{-\Delta t / \varepsilon^{2}} \frac{\mu J^{n}}{2}+\left(1-e^{-\Delta t / \varepsilon^{2}}\right) \frac{\overline{\mathbb{D}}_{+}\left(z^{n}\right)}{2}\right)\right. \\
& \left.+\mathbb{D}_{-}\left(e^{-\Delta t / \varepsilon^{2}} \frac{\left(-\mu J^{n}\right)}{2}+\left(1-e^{-\Delta t / \varepsilon^{2}}\right) \frac{\overline{\mathbb{D}}_{-}\left(z^{n}\right)}{2}\right)\right) \\
& +\Delta t\left(\sigma_{a}\left(\Theta^{n+1 / 2}-\rho^{n}\right)+S^{n}\right) \text {. }
\end{aligned}
$$

while the second step will coincide with Step 2 of Subsection 2.2 together with $\Theta^{n+1}=\Theta^{n+1 / 2}$. From here, we can write the completely relaxed scheme

$$
\begin{aligned}
J^{n+1}=e^{-\Delta t / \varepsilon^{2}} J^{n}+ & \frac{1-e^{-\Delta t / \varepsilon^{2}}}{2 \mu}\left(\overline{\mathbb{D}}_{+}\left(\rho^{n} \psi^{n}\right)-\overline{\mathbb{D}}_{-}\left(\rho^{n} \psi^{n}\right)\right) \\
+\frac{\Delta t}{\varepsilon \mu}\left[\mathbb{D}_{+}\left(e^{-\Delta t / \varepsilon^{2}} \frac{\mu J^{n}}{2}+\left(1-e^{-\Delta t / \varepsilon^{2}}\right) \frac{\overline{\mathbb{D}}_{+}\left(\rho^{n} \psi^{n}\right)}{2}\right)\right. & \left.-\mathbb{D}_{-}\left(e^{-\Delta t / \varepsilon^{2}} \frac{\left(-\mu J^{n}\right)}{2}+\left(1-e^{-\Delta t / \varepsilon^{2}}\right) \frac{\overline{\mathbb{D}}_{-}\left(\rho^{n} \psi^{n}\right)}{2}\right)\right] \\
\Theta^{n+1}= & e^{-\sigma_{a} \Delta t} \Theta^{n}+\sigma_{a}\left(1-e^{-\sigma_{a} \Delta t}\right) \rho^{n} \\
\rho^{n+1}= & \rho^{n}+\frac{\Delta t}{\mu^{2}}\left(\mathbb{D}_{+}\left(e^{-\Delta t / \varepsilon^{2}} \frac{\mu J^{n}}{2}+\left(1-e^{-\Delta t / \varepsilon^{2}}\right) \frac{\overline{\mathbb{D}}_{+}\left(\rho^{n} \psi^{n}\right)}{2}\right)\right. \\
& \left.+\mathbb{D}_{-}\left(e^{-\Delta t / \varepsilon^{2}} \frac{\left(-\mu J^{n}\right)}{2}+\left(1-e^{-\Delta t / \varepsilon^{2}}\right) \frac{\overline{\mathbb{D}}_{-}\left(\rho^{n} \psi^{n}\right)}{2}\right)\right) \\
& +\Delta t\left(\sigma_{a}\left(\Theta^{n+1}-\rho^{n}\right)+S^{n}\right) .
\end{aligned}
$$


Concerning the zeroth order closure for the Su-Olson test, we have the system

$$
\left\{\begin{array}{l}
\partial_{t} \varrho-\partial_{x}\left(\frac{\varrho}{\varepsilon} \mathbb{G}\left(\varepsilon \frac{\partial_{x} \varrho}{\varrho}\right)\right)=\sigma_{a}(\Theta-\rho)+S, \\
\partial_{t} \Theta=\sigma_{a}(\rho-\Theta),
\end{array}\right.
$$

that can be seen as the relaxation, when $\alpha$ tends to 0 , of

$$
\begin{aligned}
\partial_{t} \rho+\partial_{x} J & =\sigma_{a}(\Theta-\rho)+S, \\
\partial_{t} J+\frac{\mu^{2}}{\varepsilon^{2}} \partial_{x} \rho & =-\frac{1}{\alpha}\left[J+\frac{\rho}{\varepsilon} \mathbb{G}\left(\varepsilon \frac{\partial_{x} \rho}{\rho}\right)\right], \\
\partial_{t} \Theta & =\sigma_{a}(\rho-\Theta) .
\end{aligned}
$$

Proceeding similarly to Subsection 2.4 and as above for the first order closure, we conclude the completely relaxed scheme for the density and temperature is

$$
\begin{aligned}
\Theta^{n+1}= & e^{-\sigma_{a} \Delta t} \Theta^{n}+\sigma_{a}\left(1-e^{-\sigma_{a} \Delta t}\right) \rho^{n} . \\
\rho^{n+1}= & \rho^{n}+\Delta t\left\{\mathbb{D}_{+}\left[\frac{\rho^{n}}{2 \varepsilon \mu} \mathbb{G}\left(\frac{\varepsilon}{\mu} \frac{\overline{\mathbb{D}}_{+} \rho^{n}}{\rho^{n}}\right)\right]+\mathbb{D}_{-}\left[\frac{\rho^{n}}{2 \varepsilon \mu} \mathbb{G}\left(\frac{\varepsilon}{\mu} \frac{\overline{\mathbb{D}}_{-} \rho^{n}}{\rho^{n}}\right)\right]\right\} \\
& +\Delta t\left(\sigma_{a}\left(\Theta^{n+1}-\rho^{n}\right)+S^{n}\right),
\end{aligned}
$$

For intermediate values of the parameter $\varepsilon$, it is worth comparing the results obtained with the models described above to the simulations based on the semi-lagrangian SL-WENO scheme already discussed in Section 3. Indeed, remind that the asymptotic kinetic scheme was developed for the asymptotic limit $\varepsilon \rightarrow 0$. Similarly, the range of validity of the macroscopic models is also restricted to small $\varepsilon$ 's; furthermore, the theoretical results in [13] prove the validity of these models for density values close to constant and far from vacuum. Hence, it is worthy to compare its results to those of the previous scheme particularly for moderate values of $\varepsilon$. The SL-WENO scheme for the Su-Olson test summarizes as follows:

Step 1.- Relax $f$

$$
\partial_{t} f=\frac{1}{\varepsilon^{2}} Q(f)+\sigma_{a}(\Theta-\rho)+S
$$

Step 2.- Compute advection and relax the temperature

$$
\begin{gathered}
\partial_{t} f+\frac{v}{\varepsilon} \partial_{x} f=0 \\
\partial_{t} \Theta=\sigma_{a}(\rho-\Theta)
\end{gathered}
$$

which gives the following numerical method: 


$$
\begin{aligned}
& \text { Step 1.- Relax } f \\
& f^{n+1 / 2}=e^{-\Delta t / \varepsilon^{2}} f^{n}+\left(1-e^{-\Delta t / \varepsilon^{2}}\right)\left[\rho^{n}+\varepsilon^{2}\left(\sigma_{a}\left(\Theta^{n}-\rho^{n}\right)+S^{n}\right)\right] \\
& \Theta^{n+1 / 2}=\Theta^{n}
\end{aligned}
$$

Step 2.- Compute advection by an interpolation method and relax the temperature

$$
\begin{gathered}
f^{n+1}\left(x_{i}, v_{j}\right)=f_{\varepsilon}^{n}\left(x_{i}-\Delta t \frac{v_{j}}{\varepsilon}\right), \\
\Theta^{n+1}=e^{-\sigma_{a} \Delta t} \Theta^{n+1}+\sigma_{a}\left(1-e^{-\sigma_{a} \Delta t}\right) \rho^{n+1} .
\end{gathered}
$$

For the simulations, the source term $S(x)$ has been chosen as the characteristic function of the interval $[0,1]$ inside the total interval $[0,30]$ with $\varepsilon=0.01$ and $\varepsilon=0.26$ respectively. We refer to the results in $[43,48,5,7]$ for comparison. The solutions of the macroscopic models are computed with the complete relaxed methods $\alpha=0$ with mesh $N_{x}=256$ and $N_{v}=256$. The traditional test considers as initial data the constant equilibrium value $10^{-10}$ for $f_{0}=\rho_{0}=\Theta_{0}$. The smallness of this value makes the simulation particularly tough; hence, we also perform the computations with $f_{0}=\rho_{0}=\Theta_{0}=1$. We make different runs, with $\varepsilon$ varying from 0.026 to 0.26 . The numerical results are displayed in Figures 7 to 12 .

A first conclusion is that the SL-WENO code is highly sensitive to the changes of $\varepsilon$, see Figure 7-(i) and(j), as already seen above, see Figure 1 ; we believe that the results become relevant only for the largest values of $\varepsilon(\varepsilon=0.26, \varepsilon=1)$, see Figures $10,11,12$. The result in the case $\varepsilon=0.26$ is surprisingly close to the solution of the heat equation. This is a bit misleading since in this regime there is no reason why the heat equation can describe the dynamics of the kinetic equation well. Other tests with direct finite-differences WENO schemes as the ones used in [8] may be interesting to clarify this point, although not directly linked to the asymptotic discussion in this paper, and thus it will be treated elsewhere.

We observe that the results given by the heat equation, the two closure models and the kinetic scheme are almost undistinguishable from each other up to final time 10, for small $\varepsilon$ 's, see Figures 8, 9. Differences appear as $\varepsilon$ grows and correspond to the results in $[5,7]$. There are discrepancies between the diffusion model, the other macroscopic models and the kinetic equation, especially for earlier times. These discrepancies reduce as time grows. It is also worth pointing out that, as in $[5,7]$ and contrarily to $[43,48]$, the results are oscillation free for the first order closure, both for the density $\rho$ and the reduced flux $\varepsilon J / \rho$, which remains bounded by 1 , as expected.

The kinetic scheme is also sensitive to the variations of $\varepsilon$, particularly for the almost vanishing initial data, see Figure 7-(g) and (h). We observe 
that the results differ from the ones given by SL-WENO for $\varepsilon=0.26$ and almost vanishing initial data: the main errors appear in the regions of large gradient of the density, see Figure 10. Clearly the kinetic scheme is not well adapted for this regime for such a small initial data. However, the performances are better considering a larger initial data, since in such a case the slopes are less steep see Figures 11 and 12. Note that this test also shows the limitation of the asymptotic-induced method since when $\varepsilon$ grows we are faced with difficulties related to the maximum principle, see Remark 6. In particular, the scheme for the first order closure is not positive in the sense of [7], the computed $\varepsilon J / \rho$ can violate the limited flux condition and we are in trouble to evaluate the flux (again, increasing the initial data makes things easier). Finally, it is remarkable to observe that the first order closure results are satisfactory in all regimes. This makes this closure model really valuable. More figures are available on the URL http://diffnum.gforge.inria.fr/SU-OLSON/.

\section{Conclusion}

We have proposed new numerical schemes based on splitting techniques specifically adapted to diffusion regimes. The main idea behind this strategy is the separation between the hydrodynamic quantities and the fluctuations. Hence, the method we design is explicit, asymptotic preserving, well balanced and mass preserving thanks to a suitable treatment of the numerical boundary conditions. This approach applies equally well to the original kinetic equation and to the macroscopic models coming from closure approximations.

The numerical experiments demonstrate the abilities of the scheme to give accurate quantitative estimates of the errors made by the approximations to the kinetic equation. The first order closure is shown to be the most accurate approximation, among those we chose, for the kinetic equation in the diffusive limit. This confirms that the choice of the closure by entropy minimization principle is certainly appropriate for applications where the kinetic equation is coupled with more complex systems.

\section{ACKNOWLEDGEMENTS}

The authors acknowledge for the computational resources of the Grid' 5000 project which made the simulations possible. TG and PL thank the Centre de Recerca Matemàtica, Barcelona, Catalonia for its warm hospitality. JAC and FV thank INRIA for the invitation in Lille and the support from the Spanish DGI-MCYT/FEDER project MTM2005-08024. 


\section{REFERENCES}

[1] D. Aregba-Driollet and R. Natalini. Discrete kinetic schemes for multidimensional systems of conservation laws. SIAM J. Numer. Anal., 37(6):1973-2004 (electronic), 2000.

[2] C. Bardos, F. Golse, B. Perthame, and R. Sentis. The nonaccretive radiative transfer equations: existence of solutions and Rosseland approximation. J. Funct. Anal., 77(2):434-460, 1988.

[3] C. Bardos, R. Santos, and R. Sentis. Diffusion approximation and computation of the critical size. Trans. Amer. Math. Soc., 284(2):617-649, 1984.

[4] Y. Brenier. Systèmes hyperboliques de lois de conservation. Cours de DEA 92-93, Publ. Laboratoire d'Analyse Numérique, Université Pierre et Marie Curie. 1992.

[5] C. Buet and S. Cordier. Asymptotic preserving scheme and numerical methods for radiative hydrodynamic models. C. R. Math. Acad. Sci. Paris, 338(12):951-956, 2004.

[6] C. Buet and B. Despres. Asymptotic analysis of fluid models for the coupling of radiation and hydrodynamics. JQSRT, 85(3-4):385-418, 2004.

[7] C. Buet and B. Despres. Asymptotic preserving and positive schemes for radiation hydrodynamics. J. Comput. Phys., 215(2):717-740, 2006.

[8] J. A. Carrillo, I. M. Gamba, C.-W. Shu. Computational macroscopic approximations to the one-dimensional relaxation-time kinetic system for semiconductors. Phys. D, 146:289-306, 2000.

[9] J. A. Carrillo, Th. Goudon, and P. Lafitte. Simulations of two-phase flows involving a dispersed phase: Bubbling and flowing regimes. in preparation.

[10] J. A. Carrillo and F. Vecil. Non oscillatory interpolation methods applied to Vlasovbased models. Preprint, 2006.

[11] J. Cartier and A. Munnier. Geometric Eddington factor for radiative transfer problems. In Numerical methods for hyperbolic and kinetic problems, volume 7 of IRMA Lect. Math. Theor. Phys., pp. 271-293. Eur. Math. Soc., Zürich, 2005.

[12] P. H. Chavanis, J. Sommeria, and R. Robert. Statistical mechanics of twodimensional vortices and collisionless stellar systems. The Astrophysical J., 471:385399, 1996.

[13] J.-F. Coulombel, F. Golse, and Th. Goudon. Diffusion approximation and entropybased moment closure for kinetic equations. Asymptot. Anal., 45(1-2):1-39, 2005.

[14] J.-F. Coulombel and Th. Goudon. Entropy-based moment closure for kinetic equations: Riemann problem and invariant regions. J. Hyperbolic Diff. Eq., to appear.

[15] J. Dolbeault, P.A. Markowich, D. Ölz, and C. Schmeiser. Nonlinear diffusion as limit of kinetic equations with relaxation collision kernels. Preprint, 2006.

[16] B. Dubroca. Etude de régimes microscopiques, macroscopiques et transitionnels basés sur des équations cinétiques : modélisation et approximation numérique. Habilitation à diriger les recherches. Université Bordeaux 1, 2000.

[17] F. Filbet and E. Sonnendrücker. Comparison of Eulerian Vlasov solvers. Computer Physics Communications, 150: 247-266, 2003.

[18] F. Filbet, E. Sonnendrücker, and P. Bertrand. Conservative numerical schemes for the Vlasov equation. J. Comput. Phys., 172: 166-187, 2001.

[19] J. Fort. Information-theoretical approach to radiative transfer. Physica A, 243(34):275-303, 1997.

[20] Y. Giga and T. Miyakawa. A kinetic construction of global solutions of first order quasilinear equations. Duke Math. J., 50(2):505-515, 1983. 
[21] P. Godillon-Lafitte and Th. Goudon. A coupled model for radiative transfer: Doppler effects, equilibrium and non equilibrium diffusion asympotics. SIAM MMS, 4:12451279, 2005.

[22] F. Golse. Kinetic equations and asymptotic theory, in From kinetic to macroscopic model, pages 41-121. Appl. Math, vol. 4. Gauthier-Villars, 2000.

[23] F. Golse, S. Jin and C. D. Levermore. A domain decomposition analysis for a two-scale linear transport problem. M2AN. Mathematical Modelling and Numerical Analysis, 37(6): 869-892, 2003.

[24] L. Gosse and G. Toscani. An asymptotic-preserving well-balanced scheme for the hyperbolic heat equations. C. R. Math. Acad. Sci. Paris, 334(4):337-342, 2002.

[25] L. Gosse and G. Toscani. Asymptotic-preserving \& well-balanced schemes for radiative transfer and the Rosseland approximation. Numer. Math., 98(2):223-250, 2004.

[26] Th. Goudon and A. Mellet. On fluid limit for the semiconductors Boltzmann equation. J. Differential Equations, 189(1):17-45, 2003.

[27] Th. Goudon and F. Poupaud. Approximation by homogenization and diffusion of kinetic equations. Comm. Partial Differential Equations, 26(3-4):537-569, 2001.

[28] Th. Goudon and P. Lafitte. Splitting schemes for the simulation of non equilibrium radiative flows. Preprint, 2006.

[29] S. Jin and Z.-P. Xin. The relaxation schemes for systems of conservation laws in arbitrary space dimensions. Comm. Pure Appl. Math., 48:235-276, 1995.

[30] A. Klar. An asymptotic-induced scheme for nonstationary transport equations in the diffusive limit. SIAM J. Numer. Anal., 35(3):1073-1094 (electronic), 1998.

[31] A. Klar. An asymptotic preserving numerical scheme for kinetic equations in the low Mach number limit. SIAM J. Numer. Anal., 36(5):1507-1527 (electronic), 1999.

[32] A. Klar and A. Unterreiter. Uniform stability of a finite difference scheme for transport equations in diffusive regime. SIAM J. Numer. Anal., 40(3):891-913 (electronic), 2002.

[33] C. D. Levermore. A Chapman-Enskog approach to flux limited diffusion theory. Technical Report, Lawrence Livermore Laboratory, UCID-18229, 1979.

[34] C. D. Levermore. Moment closure hierarchies for kinetic theories. J. Statist. Phys., 83(5-6):1021-1065, 1996.

[35] C. D. Levermore. Entropy-based moment closures for kinetic equations. In Proceedings of the International Conference on Latest Developments and Fundamental Advances in Radiative Transfer (Los Angeles, CA, 1996), volume 26, pages 591-606, 1997.

[36] C. D. Levermore and W. J. Morokoff. The Gaussian moment closure for gas dynamics. SIAM J. Appl. Math., 59(1):72-96 (electronic), 1999.

[37] C. D. Levermore and G. C. Pomraning. A flux-limited diffusion theory. Astrophys. $J, 248: 321-334,1981$.

[38] P.-L. Lions, B. Perthame, and P. Souganidis. Existence and stability of entropy solutions for the hyperbolic systems of isentropic gas dynamics in Eulerian and Lagrangian coordinates. Comm. Pure Appl. Math., 49(6):599-638, 1996.

[39] P.-L. Lions, B. Perthame, and E. Tadmor. Kinetic formulation of the isentropic gas dynamics and p-systems. Comm. Math. Phys., 163(2):415-431, 1994.

[40] P.-L. Lions and G. Toscani. Diffuse limit for finite velocity Boltzmann kinetic models. Rev. Mat. Ib., 13:473-513, 1997. 
[41] G. Naldi, L. Pareschi, and G. Toscani. Relaxation schemes for partial differential equations and applications to degenerate diffusion problems. Surveys Math. Indust., 10(4):315-343, 2002.

[42] R. Natalini. A discrete kinetic approximation of entropy solutions to multidimensional scalar conservation laws. J. Differential Equations, 148(2):292-317, 1998.

[43] G.L. Olson, L.H. Auer, and M.L. Hall. Diffusion, P1, and other approximation of radiation transport. JQSRT, 64:619-634, 2000.

[44] B. Perthame. Kinetic formulation of conservation laws, Volume 21 of Oxford Lecture Series in Mathematics and its Applications. Oxford University Press, Oxford, 2002.

[45] B. Perthame and E. Tadmor. A kinetic equation with kinetic entropy functions for scalar conservation laws. Comm. Math. Phys., 136(3):501-517, 1991.

[46] C.-W. Shu. Essentially non-oscillatory and weighted essentially non-oscillatory schemes for hyperbolic conservation laws. In Advanced numerical approximation of nonlinear hyperbolic equations (Cetraro, 1997), volume 1697 of Lecture Notes in Math., pages 325-432. Springer, Berlin, 1998.

[47] C.-W. Shu and S. Osher. Efficient implementation of essentially nonoscillatory shockcapturing schemes. J. Comput. Phys., 77(2):439-471, 1988.

[48] B. Su and G.L. Olson. Analytical benchmark for non-equilibrium radiative transfer in anisotropically scattering medium. Annals of Nuclear Energy, 24(13):1035-1055, 1997.

[49] X. Yang, F. Golse, Z. Huang, and S. Jin. Numerical study of a domain decomposition method for a two-scale linear transport equation. Networks and Heterogenous Media, 1(1): 143-166, 2006.

(1) ICREA And Departament de Matemàtiques, Universitat Autònoma de Barcelona, E-08193 Bellaterra, Spain.

E-mail address: carrillo@mat.uab.es

(2) Team SIMPAF-INRIA Futurs Laboratoire Paul Painlevé, UMR 8524 CNRSUniversité des Sciences et Technologies de Lille, Cité Scientifique, F-59655 Villeneuve d'AscQ Cedex, France.

E-mail address: thierry.goudon, pauline.lafitte@univ-lille1.fr

(3) Departament de Matemàtiques, Universitat Autònoma de Barcelona, E08193 Bellaterra, Spain.

E-mail address: fvecil@mat.uab.es 


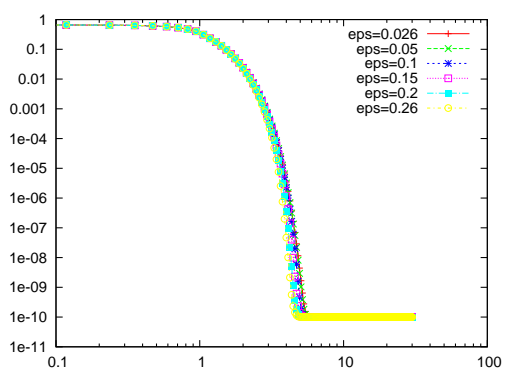

(a) Oth order model

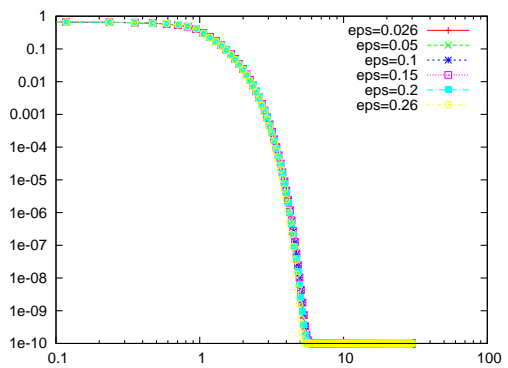

(c) 1st order model

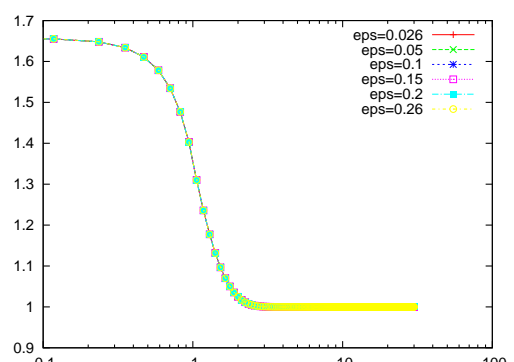

(b) 0th order model

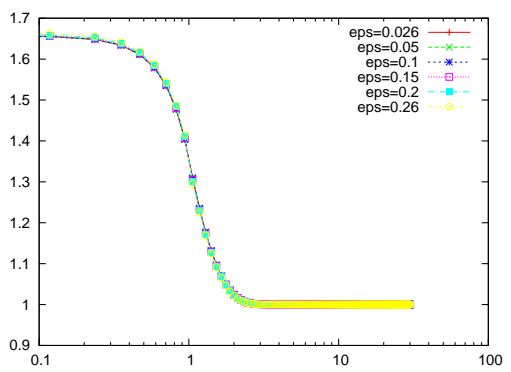

(d) 1st order model

Figure 7. Su-Olson test: Comparison of the density $\rho$ computed by the different models as $\varepsilon$ varies at time $t=1$. From top to bottom: 0th order model, 1st order model, heat equation, kinetic asymptotic-induced model, SL-WENO scheme. Left column: results in log-log scale for the initial data $f_{0}=\rho_{0}=\Theta_{0}=10^{-10}$; Right column: results in semi-log scale initial data $f_{0}=\rho_{0}=\Theta_{0}=1$. 


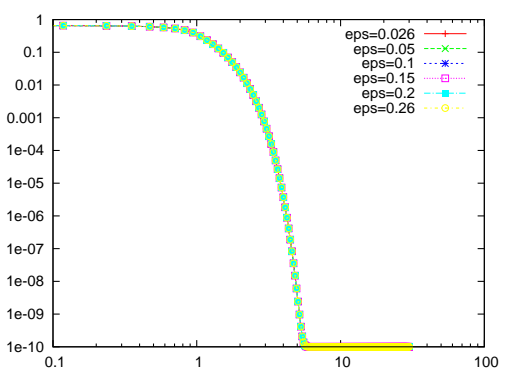

(e) Heat

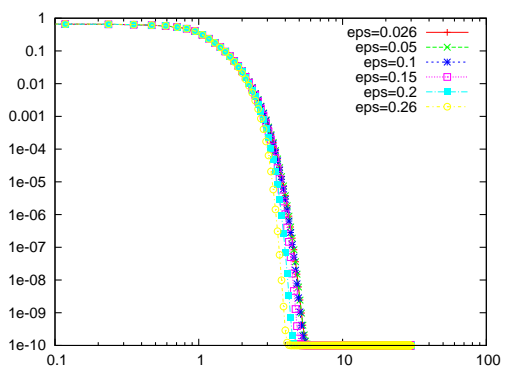

(g) Kinetic

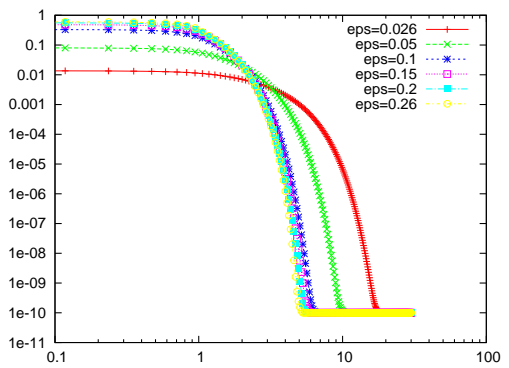

(i) SL-WENO

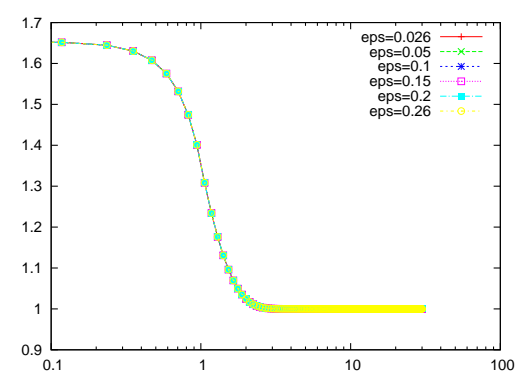

(f) Heat

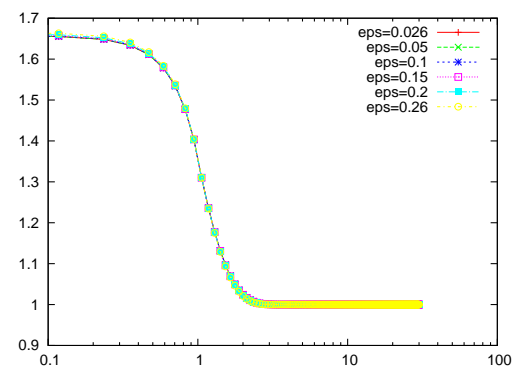

(h) Kinetic

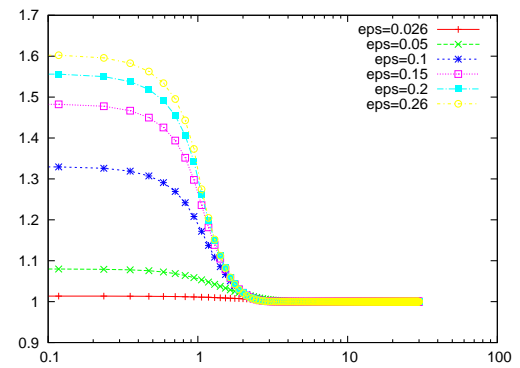

(j) SL-WENO

Figure 7. Su-Olson test: Comparison of the density $\rho$ computed by the different models as $\varepsilon$ varies at time $t=1$ (continued). 

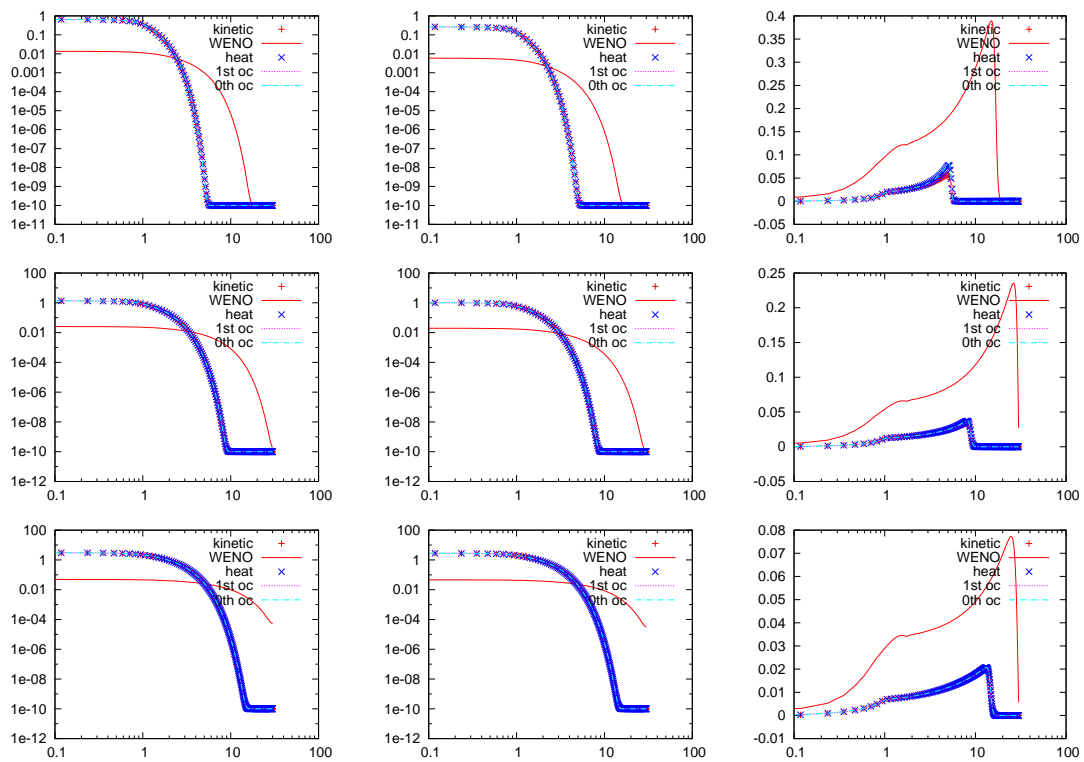

Figure 8. Su-Olson test: Left column: comparison of densities $\rho$; middle column: comparison of temperatures $\Theta$; right column : comparison of reduced fluxes $\varepsilon J / \rho$ in log-log scales for the solutions after time 1, 3 and 10 time units respectively (from top to bottom) computed with the kinetic, the heat equation, the first and the zeroth order closure methods for $\varepsilon=0.026$. The initial data is $f_{0}=\rho_{0}=\Theta_{0}=$ $10^{-10}$. 

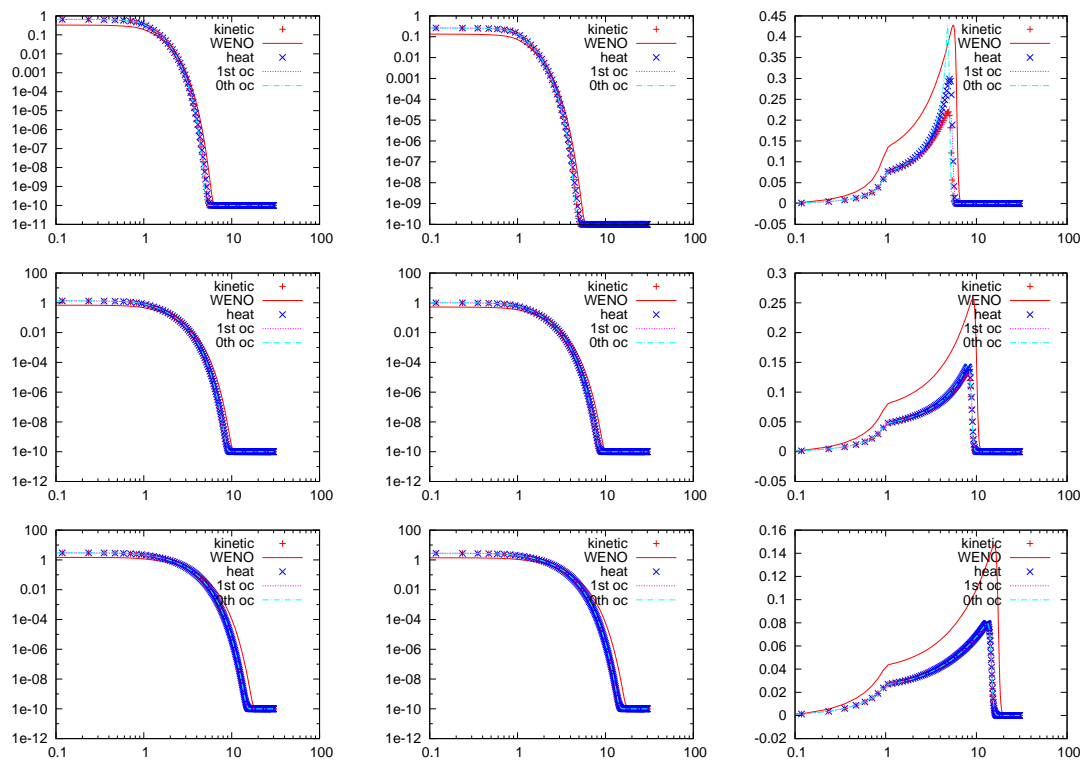

Figure 9. Su-Olson test: Left column: comparison of densities $\rho$; middle column: comparison of temperatures $\Theta$; right column : comparison of reduced fluxes $\varepsilon J / \rho$ in log-log scales for the solutions after time 1, 3 and 10 time units respectively (from top to bottom) computed with the kinetic, the heat equation, the first and the zeroth order closure methods for $\varepsilon=0.1$. The initial data is $f_{0}=\rho_{0}=\Theta_{0}=$ $10^{-10}$. 

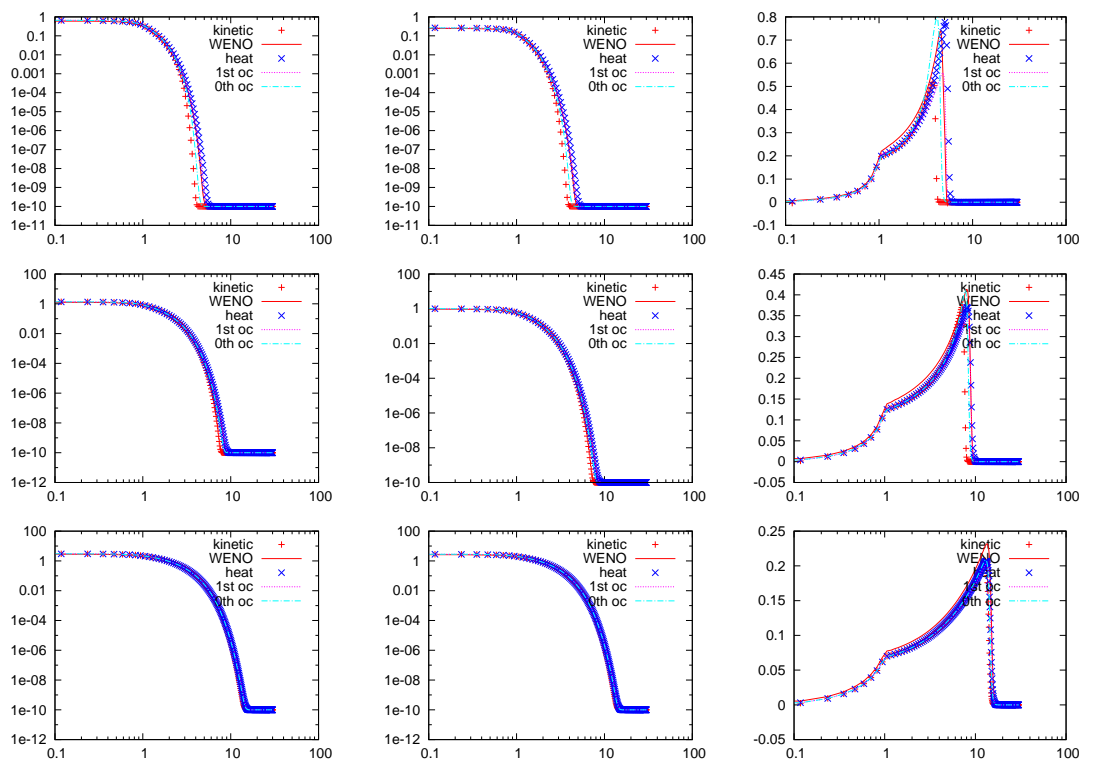

Figure 10. Su-Olson test: Left column: comparison of densities $\rho$; middle column: comparison of temperatures $\Theta$; right column : comparison of reduced fluxes $\varepsilon J / \rho$ in $\log$-log scales for the solutions after time 1,3 and 10 time units respectively (from top to bottom) computed with the kinetic, the heat equation, the first and the zeroth order closure methods for $\varepsilon=0.26$. The initial data is $f_{0}=\rho_{0}=$ $\Theta_{0}=10^{-10}$. 

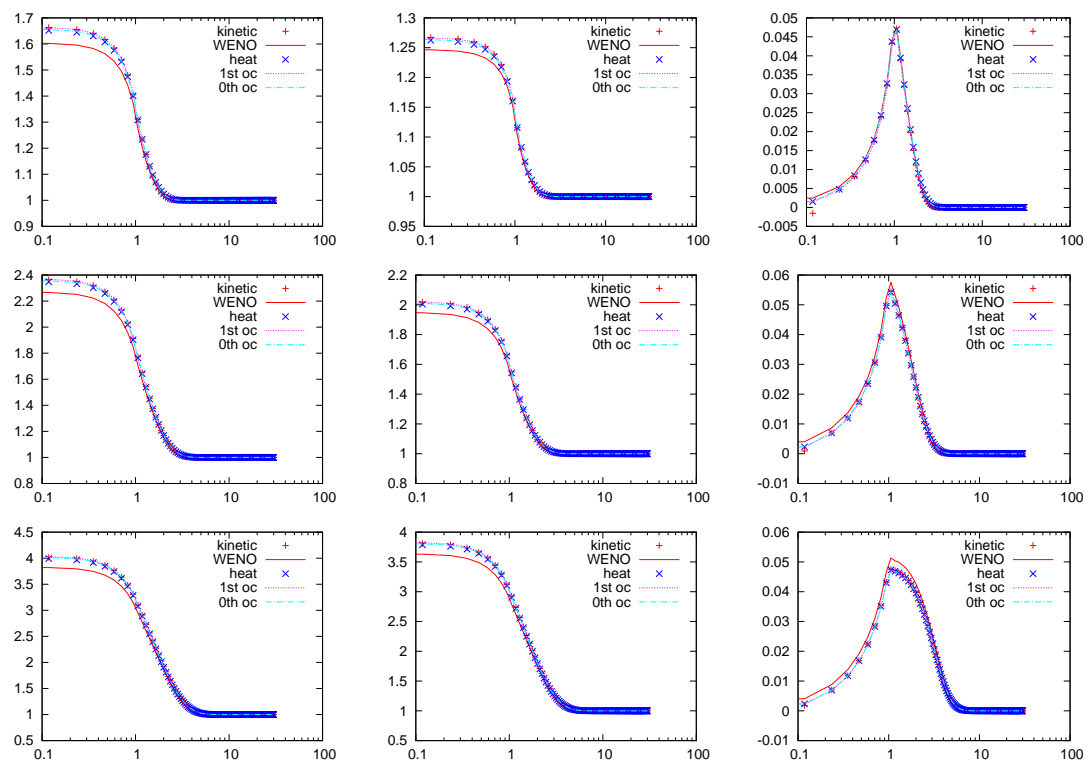

Figure 11. Su-Olson test: Left column: comparison of densities $\rho$; middle column: comparison of temperatures $\Theta$; right column : comparison of reduced fluxes $\varepsilon J / \rho$ in $\log$-log scales for the solutions after time 1, 3 and 10 time units respectively (from top to bottom) of with the kinetic, the heat equation, the first and the zeroth order closure methods for $\varepsilon=0.26$. The initial data is $f_{0}=\rho_{0}=\Theta_{0}=1$. 